\title{
Type la Supernova Discoveries atz> 1 from the Hubble Space Telescope: Evidence for Past Deceleration and Constraints on Dark Energy Evolution
}

\section{Citation}

Riess, Adam G., Louis\#Gregory Strolger, John Tonry, Stefano Casertano, Henry C. Ferguson, Bahram Mobasher, Peter Challis, et al. 2004. "Type la Supernova Discoveries Atz> 1 from the Hubble Space Telescope: Evidence for Past Deceleration and Constraints on Dark Energy Evolution." The Astrophysical Journal 607 (2): 665-87. https://doi.org/10.1086/383612.

\section{Permanent link}

http://nrs.harvard.edu/urn-3:HUL.InstRepos:41399911

\section{Terms of Use}

This article was downloaded from Harvard University's DASH repository, and is made available under the terms and conditions applicable to Other Posted Material, as set forth at http:// nrs.harvard.edu/urn-3:HUL.InstRepos:dash.current.terms-of-use\#LAA

\section{Share Your Story}

The Harvard community has made this article openly available.

Please share how this access benefits you. Submit a story.

\section{Accessibility}




\title{
TYPE Ia SUPERNOVA DISCOVERIES AT $z>1$ FROM THE HUBBLE SPACE TELESCOPE: EVIDENCE FOR PAST DECELERATION AND CONSTRAINTS ON DARK ENERGY EVOLUTION ${ }^{1}$
}

\author{
Adam G. Riess, ${ }^{2}$ Louis-Gregory Strolger, ${ }^{2}$ John Tonry, ${ }^{3}$ Stefano Casertano, ${ }^{2}$ Henry C. Ferguson, ${ }^{2}$ Bahram Mobasher, ${ }^{2}$ \\ Peter Challis, ${ }^{4}$ Alexei V. Filippenko, ${ }^{5}$ Saurabh Jha, ${ }^{5}$ Weidong Li, ${ }^{5}$ Ryan Chornock, ${ }^{5}$ Robert P. Kirshner, ${ }^{4}$ \\ Bruno Leibundgut, ${ }^{6}$ Mark Dickinson, ${ }^{2}$ Mario Livio, ${ }^{2}$ Mauro Giavalisco, ${ }^{2}$ \\ Charles C. Steidel, ${ }^{7}$ Txitxo Benítez, ${ }^{8}$ and Zlatan Tsvetanov ${ }^{8}$ \\ Received 2004 January 20; accepted 2004 February 16
}

\begin{abstract}
We have discovered 16 Type Ia supernovae (SNe Ia) with the Hubble Space Telescope (HST) and have used them to provide the first conclusive evidence for cosmic deceleration that preceded the current epoch of cosmic acceleration. These objects, discovered during the course of the GOODS ACS Treasury program, include 6 of the 7 highest redshift SNe Ia known, all at $z>1.25$, and populate the Hubble diagram in unexplored territory. The luminosity distances to these objects and to 170 previously reported $\mathrm{SNe}$ Ia have been determined using empirical relations between light-curve shape and luminosity. A purely kinematic interpretation of the SN Ia sample provides evidence at the greater than $99 \%$ confidence level for a transition from deceleration to acceleration or, similarly, strong evidence for a cosmic jerk. Using a simple model of the expansion history, the transition between the two epochs is constrained to be at $z=0.46 \pm 0.13$. The data are consistent with the cosmic concordance model of $\Omega_{M} \approx 0.3, \Omega_{\Lambda} \approx 0.7\left(\chi_{\mathrm{dof}}^{2}=1.06\right)$ and are inconsistent with a simple model of evolution or dust as an alternative to dark energy. For a flat universe with a cosmological constant, we measure $\Omega_{M}=0.29 \pm_{0.03}^{0.05}$ (equivalently, $\Omega_{\Lambda}=0.71$ ). When combined with external flat-universe constraints, including the cosmic microwave background and large-scale structure, we find $w=-1.02 \pm_{0.19}^{0.13}$ (and $w<-0.76$ at the $95 \%$ confidence level) for an assumed static equation of state of dark energy, $P=w \rho c^{2}$. Joint constraints on both the recent equation of state of dark energy, $w_{0}$, and its time evolution, $d w / d z$, are a factor of $\sim 8$ more precise than the first estimates and twice as precise as those without the SNe Ia discovered with HST. Our constraints are consistent with the static nature of and value of $w$ expected for a cosmological constant (i.e., $w_{0}=-1.0$, $d w / d z=0)$ and are inconsistent with very rapid evolution of dark energy. We address consequences of evolving dark energy for the fate of the universe.
\end{abstract}

Subject headings: cosmology: observations — distance scale — galaxies: distances and redshifts — supernovae: general

On-line material: machine-readable tables

\section{INTRODUCTION}

Observations of Type Ia supernovae (SNe Ia) at redshift $z<1$ provide startling and puzzling evidence that the expansion of the universe at the present time appears to be accelerating, behavior attributed to "dark energy" with negative pressure (Riess et al., 1998; Perlmutter et al. 1999; for reviews, see Riess 2000; Filippenko 2001, 2004; Leibundgut 2001). Direct evidence comes from the apparent faintness of $\mathrm{SNe}$ Ia at $z \approx 0.5$. Recently expanded samples of SNe Ia have

\footnotetext{
${ }^{1}$ Based on observations with the NASA/ESA Hubble Space Telescope, obtained at the Space Telescope Science Institute, which is operated by AURA, Inc., under NASA contract NAS5-26555.

2 Space Telescope Science Institute, 3700 San Martin Drive, Baltimore, MD 21218.

${ }^{3}$ Institute for Astronomy, University of Hawaii, 2680 Woodlawn Drive, Honolulu, HI 96822.

${ }^{4}$ Harvard-Smithsonian Center for Astrophysics, 60 Garden Street, Cambridge, MA 02138.

5 Department of Astronomy, 601 Campbell Hall, University of California, Berkeley, CA 94720-3411.

${ }^{6}$ European Southern Observatory, Karl-Schwarzschild-Strasse 2, D-85748 Garching, Germany.

7 Department of Astronomy, 105-24, California Institute of Technology, Pasadena, CA 91125.

${ }^{8}$ Department of Physics and Astronomy, Johns Hopkins University, Baltimore, MD 21218.
}

reinforced the statistical significance of this result (Knop et al. 2003), while others have also extended the SN Ia sample to $z \approx 1$ (Tonry et al. 2003; Barris et al. 2004). Observations of large-scale structure (LSS), when combined with measurements of the characteristic angular size of fluctuations in the cosmic microwave background (CMB), provide independent (although indirect) evidence for a dark energy component (e.g., Spergel et al. 2003). An independent, albeit more tentative, investigation via the integrated Sachs-Wolfe (ISW) effect also provides evidence for dark energy (Scranton et al. 2003). The magnitude of the observed acceleration was not anticipated by theory and continues to defy a post facto explanation. Candidates for the dark energy include Einstein's cosmological constant $\Lambda$ (with a phenomenally small value), evolving scalar fields (modern cousins of the inflation field; Caldwell et al. 1998; Peebles \& Ratra 2003), and a weakening of gravity in our $3+1$ dimensions by leaking into the higher dimensions required in string theories (Deffayet et al. 2002). These explanations bear so greatly on fundamental physics that observers have been stimulated to make extraordinary efforts to confirm the initial results on dark energy, test possible sources of error, and extend our empirical knowledge of this newly discovered component of the universe.

Astrophysical effects could imitate the direct evidence from $\mathrm{SNe}$ Ia for an accelerating universe. A pervasive screen of 
TABLE 1

DisCovery DatA

\begin{tabular}{|c|c|c|c|c|}
\hline SN & Nickname & UT Date & SN $\alpha(J 2000.0)$ & $\mathrm{SN} \delta(\mathrm{J} 2000.0)$ \\
\hline $2002 \mathrm{fw}$ & Aphrodite & Sep 19.86 & 033237.52 & -274646.6 \\
\hline $2002 \mathrm{fx}$ & Athena & Sep 20.84 & 033206.80 & -274434.4 \\
\hline $2002 \mathrm{hp}$ & Thoth & Nov 1.51 & 033224.79 & -274617.8 \\
\hline $2002 \mathrm{hr} \ldots \ldots \ldots \ldots \ldots \ldots \ldots \ldots$ & Isis & Nov 1.64 & 033222.57 & -274152.2 \\
\hline $2002 \mathrm{kc}$ & Bilbo & Dec 21.50 & 033234.72 & -273958.3 \\
\hline $2002 \mathrm{kd} \ldots \ldots \ldots \ldots . . . .$. & Frodo & Dec 21.64 & 033222.34 & -274426.9 \\
\hline $2002 \mathrm{ki} \ldots \ldots \ldots \ldots . . . .$. & Nanna & Nov 22.69 & 123728.35 & +622040.0 \\
\hline 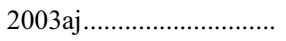 & Inanna & Feb 3.19 & 033244.33 & -275506.4 \\
\hline 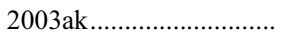 & Gilgamesh & Feb 3.19 & 033246.90 & -275449.4 \\
\hline 2003az ............................. & Torngasek & Feb 20.91 & 123719.67 & +62 1837.5 \\
\hline 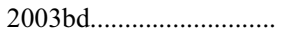 & Anguta & Feb 21.95 & 123725.06 & +62 1317.5 \\
\hline 2003be................................... & Qiqirn & Feb 22.08 & 123625.97 & +620655.6 \\
\hline 2003 dy .................................. & Borg & Apr 4.67 & 123709.16 & +62 1129.0 \\
\hline 2003XX & Vilas & Apr 4.67 & 123729.00 & +62 1127.8 \\
\hline $2003 \mathrm{eb}$ & McEnroe & Apr 5.65 & 123715.18 & +62 1334.6 \\
\hline 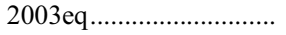 & Elvis & May 24.7 & 123748.34 & +62 1335.3 \\
\hline 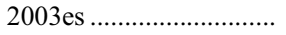 & Ramone & May 25.5 & 123655.39 & +621311.9 \\
\hline
\end{tabular}

gray dust could dim SNe Ia with little telltale reddening (Aguirre 1999a, 1999b). Luminosity evolution could corrupt the measurements if SNe Ia at $z \approx 0.5$ are intrinsically fainter than their low-redshift counterparts. To date, no evidence for an astrophysical origin of the apparent faintness of SNe Ia has been found (Riess 2000; Coil et al. 2000; Leibundgut 2001; Sullivan et al. 2003). However, given the significance of the putative dark energy and the unique ability of $\mathrm{SNe}$ Ia to illuminate it, we need a more definitive test of the hypothesis that supernovae at $z \sim 0.5$ are intrinsically dimmer, or dimmed by absorption.

If cosmic acceleration is the reason why $\mathrm{SNe}$ Ia are dimmer at $z \sim 0.5$, then we expect cosmic deceleration at $z>1$ to reverse the sign of the observed effect. The combination of recent acceleration and past deceleration is a clear signature of a mixed dark matter and dark energy universe and one that is readily distinguishable from simple astrophysical dimming (Filippenko \& Riess 2001).

Furthermore, assuming that $\mathrm{SNe}$ Ia at $z>1$ continue to trace the cosmological world model, measurements of SNe Ia in the next redshift octave provide the unique ability to discriminate between a static and evolving dark energy equation of state. This would provide a vital clue to distinguish a cosmological constant from other forms of dark energy that change with time.

Ground-based efforts to look for past deceleration with $\mathrm{SNe}$ Ia have offered hints of the effect, but ultimately they have suffered from insufficient signal-to-noise ratios (S/Ns) (Tonry et al. 2003; Barris et al. 2004). Discovering, confirming, and then monitoring transients at $I \approx 25 \mathrm{mag}$ on the bright sky is challenging even with the largest telescopes and the best conditions. A single SN Ia at $z \approx 1.7, \mathrm{SN} 1997 \mathrm{ff}$, discovered with WFPC2 on the Hubble Space Telescope (HST) (Gilliland et al. 1999), provided a hint of past deceleration; however, inferences drawn from a single SN Ia, while plausible, are not robust (Riess et al. 2001; Benítez et al. 2002; Mortsell et al. 2001, hereafter MGG2001).

To study the early expansion history of the universe, we initiated the first systematic, space-based search and follow-up effort to collect SNe Ia at $z>1$, carried out in conjunction with the Great Observatories Origins Deep Survey (GOODS) Treasury program (Giavalisco et al. 2004) conducted with the Advanced Camera for Surveys (ACS) aboard HST. (The ability to detect $\mathrm{SNe}$ at $z>1$ with the Space Telescope was an application first envisioned during its planning; Tammann 1979; Colgate 1979.) A separate "piggyback" program was utilized to obtain target of opportunity (ToO) follow-up HST observations of the SNe Ia with ACS and NICMOS (the NearInfrared Camera and Multi-Object Spectrograph). Elsewhere we present a color-based method for discrimination of SNe Ia at $z>1$ from other transients (Riess et al. 2004) and the full harvest of the SN survey (L.-G. Strolger et al. 2004, in preparation). We present the follow-up spectroscopy and photometry of $16 \mathrm{SNe}$ Ia in $\S 2$, light-curve analysis in $\S 3$, cosmological tests and constraints in $\S 4$, and a discussion and summary in $\S \S 5$ and 6 , respectively.

\section{TARGET OF OPPORTUNITY FOLLOW-UP: LIGHT CURVES AND SPECTRA}

\subsection{Follow-up}

The methods and criteria that we used to search the GOODS ACS Treasury data for SNe are described by L.-G. Strolger et al. (2004, in preparation) and are based on image subtraction (Perlmutter et al. 1997; Schmidt et al. 1998). Strolger et al. provide the parameters of the search, including search depth, efficiency, timing, and false-positive discrimination, as well as a list of all detected SNe. Briefly, our search was conducted in the F850LP ( $Z$ band) to an effective limit of $\sim 26.0$ (Vega) magnitude covering 0.1 square degrees in 5 epochs (at intervals of $\sim 45$ days). Our limiting magnitude was 1-2 mag fainter than the expected peak of a SN Ia over the target range of $1<z<$ 1.6; therefore, $\mathrm{SNe}$ Ia that we collected (whose intrinsic dispersion is expected to be $<0.2 \mathrm{mag}$ ) would not preferentially be selected from the bright tail of their intrinsic distribution. In Table 1 we provide discovery data for the SNe Ia reported here.

Our $\mathrm{ToO}$ candidates were generally too faint to anticipate useful spectral discrimination from the ground; it was therefore necessary to initially identify SNe Ia photometrically. To discriminate $\mathrm{SNe}$ Ia at $z>1$ from $\mathrm{SNe}$ II and from SNe I at lower redshifts, we used a combination of photometric redshifts of the host galaxies (with nine passbands) and rest-frame ultraviolet (UV) colors; see Riess et al. (2004) for details. A comparison of the photometric redshifts available at the start of the survey with spectroscopic redshifts obtained thereafter 


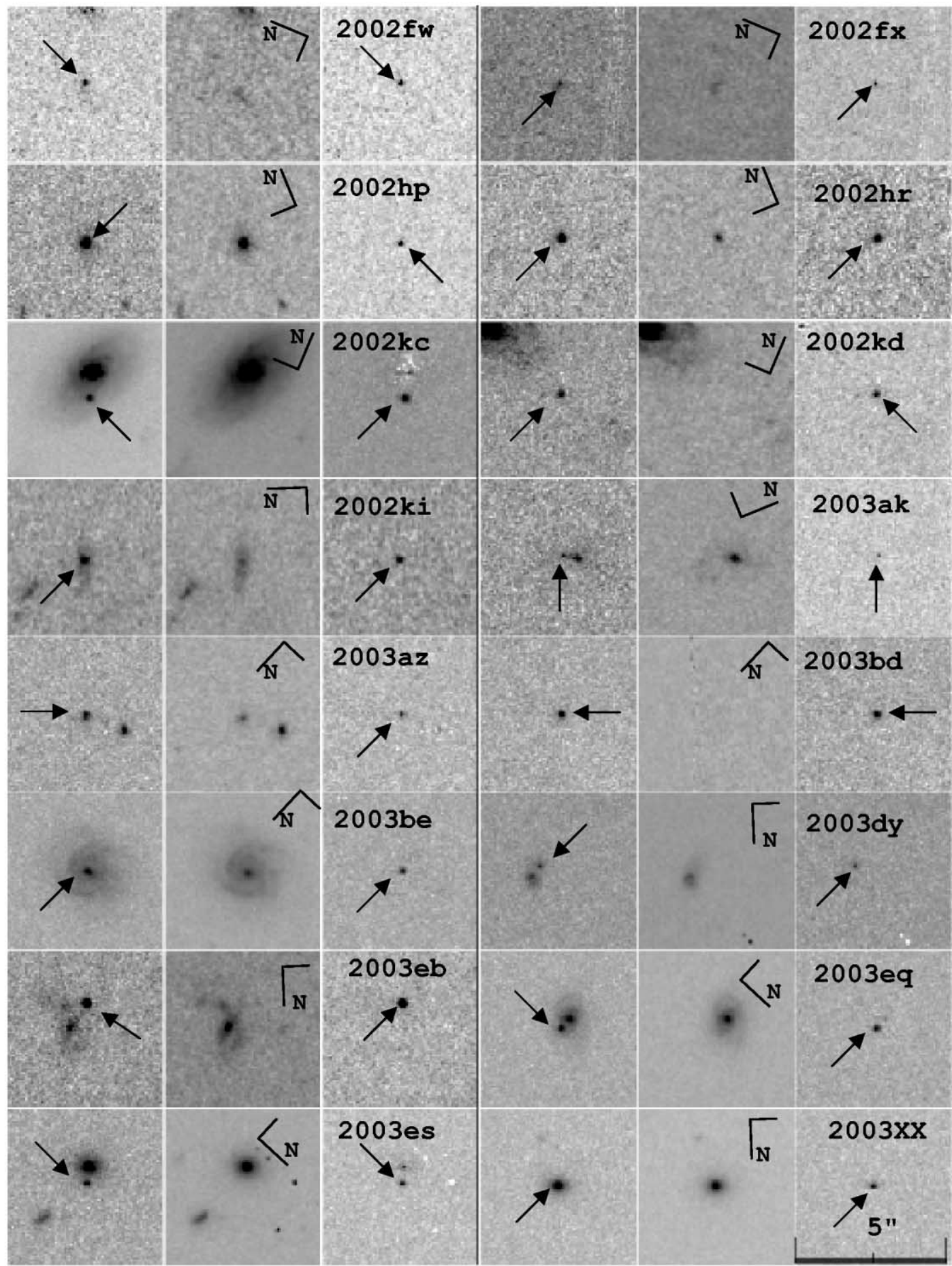

FIG. 1.-Discovery-image sections from ACS F850LP images around each SN. Panels on the left and middle show the discovery epoch and the preceding (template) epoch, respectively. The panels on the right show the results of the subtraction (discovery epoch minus template). Arrows indicate position of the SN. Image scales and orientations are given.

yields an rms of 0.05 for the quantity $\left(z_{\text {phot }}-z_{\text {spec }}\right) /\left(1+z_{\text {spec }}\right)$ for 25 hosts (with a $4 \sigma$ outlier; see L.-G. Strolger et al. 2004, in preparation).

We selected nine individual candidates for subsequent $\mathrm{ToO}$ observations (including one pair of targets observed within the same ACS field). In addition to these primary targets, judicious positioning of the follow-up fields provided serendipitous monitoring of four additional high-redshift SN Ia candidates. For four more $\mathrm{SNe}$ Ia, the periodic imaging of the GOODS survey (sometimes augmented with a few ground- based observations) provided sufficient characterization of their light curves. Subsections of the discovery and prediscovery images, as well as their difference (centered on each SN Ia), are shown in Figure 1.

The great benefit of HST observations-dramatically reduced sky noise-is only fully realized if operational constraints can be overcome. To get the full advantage of HST, the supernova search needs to be completed, the best targets selected, the spectra taken, and the photometric follow-up set in motion in 2 weeks. Otherwise, the peak of the light curve will 
be missed, the decline rate of the object will not be well measured, and as the objects fade, the spectra will become too difficult to obtain. Our challenge was to do all this without using highly disruptive and inefficient " 24 hour" ToOs (for which the HST weekly schedule is immediately interrupted, wasting precious observing time). To achieve our goals, each GOODS epoch was scheduled during a 3-4 day interval immediately preceding the weekly deadline for a ToO activation. This allowed the ToO observing to be scheduled and uploaded to the spacecraft for the following week's observations without delay. By adopting this prescription we were able to achieve a time interval of typically 9-11 days between the discovery and first follow-up observation, a span of less than 5 days in the rest frame of an SN at $z \gtrsim 1$.

After discovery and photometric screening, we developed a follow-up plan that was appropriate for our best estimate of the SN redshift. ACS and the F850LP filter were typically used for 6-8 epochs to obtain a rest-frame $B$-band or $U$-band light curve for SNe Ia at $z>1$ extending to $\sim 20$ rest-frame days past maximum brightness. This strategy utilized results from S. Jha et al. (2004a, in preparation), who demonstrated the utility of a large sample of $U$-band light curves for constraining the lightcurve shape parameter and phasing of the optical light curves. To provide rest-frame optical zero points, where $\mathrm{SNe}$ Ia are best calibrated, we used NICMOS Camera 2. SNe expected to be at $1.0<z<1.3$ were imaged with NICMOS Camera 2 and F110W. SNe expected to be at $z>1.3$ were imaged with both F110W and F160W. The one exception was SN 2002ki, for which a ground-based spectrum yielded $z=1.14$ from narrow, host-galaxy [O II] emission, and $\mathrm{F} 160 \mathrm{~W}$ was used to sample the second maximum in the rest-frame infrared (IR) to use as a secondary means of classification.

ACS coupled with a grism filter (G800L) provides slitless spectroscopy over the entire wide-field camera (WFC) field of view. For obtaining spectra of high-redshift SNe Ia, this mode of observing has noteworthy advantages and disadvantages, as compared to spatial-blocking spectroscopy with STIS on HST or from the ground with a large-aperture telescope. The primary advantage of ACS grism spectroscopy is its efficiency. ACS is the most efficient camera to fly on HST because of its rationed, silver-coated reflections. Importantly, slitless spectroscopy with the grism retains the efficiency of HST resolution along one spatial direction, dispersing the light of a pointspread function (PSF) over few sky pixels. Another advantage attuned to identifying high-redshift SNe Ia is the relatively low sky brightness between 0.8 and $1 \mu \mathrm{m}$ from the vantage point of $H S T$.

Using the HST grism has disadvantages as well. $H S T$ is a factor of 3-4 smaller in size than the largest ground-based optical telescopes. Moreover, the grism disperses a large spectral range of the sky onto the position of the SN. For G800L, wavelengths shorter than $\sim 5500 \AA$ are blocked, so the total sky counts are only $\sim 50 \%$ greater (hence $\sim 25 \%$ more noise) than for the $I$ band $(\mathrm{F} 814 \mathrm{~W})$. A more troublesome feature of grism observations is the superposition of multiple sources from multiple diffraction orders on the same sky pixels. Careful consideration must be given to possible contamination of an SN spectrum by nearby sources, especially the host galaxy (although this effect can be mitigated by judicious choice of the telescope roll angle). The ACS grism also is limited in resolution to $R=\lambda / \Delta \lambda=200$. However, this resolution is well matched to measure $\mathrm{SNe}$ Ia whose blended absorption features are broadened by ejecta dispersions of $10^{4} \mathrm{~km} \mathrm{~s}^{-1}$. Serendipitous spectra of SNe Ia obtained during
ACS commissioning and reported by Blakeslee et al. (2003) provided the first in-orbit examples at high redshift (specifically, SN 2002dc at $z=0.47$ and SN 2002dd at $z=0.95$ ).

We employed the ACS grism for our ToOs when we expected to obtain a sufficient $\mathrm{S} / \mathrm{N}$ for classification and redshift determination in fewer than 8 orbits of integration time and without detrimental contamination. These included $\mathrm{SNe}$ $2002 \mathrm{fw}, 2003 \mathrm{az}, 2003 \mathrm{dy}, 2003 \mathrm{es}$, and 2003eq, as well as the neighboring SN 2003eb. Spectra of other SNe or host galaxies were obtained with ground-based telescopes (Table 3), including the VLT, Magellan, Keck II with NIRSPEC (McLean et al. 1998), and especially Keck I with LRIS (Oke et al. 1995).

\subsection{Photometry}

After the search phase, all images were reprocessed using up-to-date reference files and the CALACS pipeline in the STSDAS package in IRAF. ${ }^{\text {}}$ This procedure includes "standard" rectifications for the camera gain, overscan, spatial bias, dark current, and flat-fielding. Because of the significant geometric distortion of the ACS WFC (the cost of minimizing reflections), we applied the drizzle algorithm (Fruchter \& Hook 1997) in the Multidrizzle software package (A. Koekemoer et al. 2004, in preparation). Because ACS WFC images are undersampled at wavelengths shortward of $11000 \AA$, a better sampled and more precise SN PSF can be obtained by "drizzling" (i.e., resampling and combining) the images at a pixel scale finer than the physical ACS WFC size of 0 .'05 pixel $^{-1}$. However, such improvements can only be realized with well-dithered images. The relative size of the dither was measured for each frame using source catalogs. Nearly all of the F850LP images in the survey and its followup were obtained at four independent dither positions and were subsequently resampled to $0.033 \mathrm{pixel}^{-1}$. Imaging in F775W and F606W utilized only two dither points, and the physical pixel scale was maintained.

For NICMOS reductions the CALNICA pipeline in the STSDAS package in IRAF was used to provide calibrated frames. Then the well-dithered frames were drizzled to half the physical pixel scale of Camera 2, i.e., 0.038 pixel $^{-1}$. The size of each frame's dither was determined by cross-correlation of common sources.

The phasing of GOODS (spanning more than 200 days) provided additional observations of the host galaxies with negligible SN light to serve as subtraction templates for the ACS data. Subsequent light-curve fitting was used to estimate the expected brightness of the SN at the time of each GOODS epoch. Epochs with negligible contamination from SN light were combined to obtain deep subtraction templates. By the last epoch of GOODS, SNe discovered in the first search had faded sufficiently ( $\sim 2$ months past maximum and 3-4 mag below peak) to provide useful templates. For $\mathrm{SNe}$ found in all subsequent searches, the initial survey epochs provided the components of deep templates.

Because of the remarkable stability of rectified ACS images, it was generally not necessary to "blur" (i.e., convolve) images to match the PSFs at different epochs (a conclusion reached by fixed-aperture tests on field stars in successive epochs). Subtractions free from host contamination were obtained by flux-conserving registration of the templates to the follow-up frames. The two exceptions were SN $2002 \mathrm{hp}$

\footnotetext{
${ }^{9}$ IRAF is distributed by the National Optical Astronomy Observatory, which is operated by the Association of Universities for Research in Astronomy, Inc., under cooperative agreement with the National Science Foundation.
} 
TABLE 2

SN Ia IMAGING

\begin{tabular}{|c|c|c|c|c|c|}
\hline $\mathrm{JD}-2,450,000$ & Filter & Vega Magnitude & Epoch (Rest) & Passbands for Transformation & $K$-Correction \\
\hline \multicolumn{6}{|c|}{ SN $2002 \mathrm{fx}$} \\
\hline $495.00 \ldots \ldots \ldots \ldots \ldots . . .$. & F775W & $28.0(1.0)$ & -13.5 & $\mathrm{~F} 775 \mathrm{~W} \rightarrow U$ & $0.001(0.06)$ \\
\hline $537.80 \ldots \ldots \ldots \ldots \ldots . . .$. & F775W & $27.07(0.25)$ & 4.2 & $\mathrm{~F} 775 \mathrm{~W} \rightarrow U$ & $0.33(0.05)$ \\
\hline $580.00 \ldots \ldots \ldots \ldots \ldots \ldots$ & F775W & $28.97(1.00)$ & 21.8 & $\mathrm{~F} 775 \mathrm{~W} \rightarrow U$ & $0.81(0.02)$ \\
\hline 490.39.................. & F850LP & $27.48(0.45)$ & -15.5 & $\mathrm{~F} 850 \mathrm{LP} \rightarrow B$ & $-1.48(0.02)$ \\
\hline $537.79 \ldots \ldots \ldots \ldots \ldots \ldots$ & F850LP & $25.17(0.07)$ & 4.2 & $\mathrm{~F} 850 \mathrm{LP} \rightarrow B$ & $-1.16(0.03)$ \\
\hline $580.49 \ldots \ldots \ldots \ldots \ldots \ldots$ & F850LP & $27.07(0.27)$ & 22.0 & $\mathrm{~F} 850 \mathrm{LP} \rightarrow B$ & $-0.79(0.03)$ \\
\hline
\end{tabular}

Notes.-Uncertainties are listed in parentheses. Table 2 is published in its entirety in the electronic edition of the Astrophysical Journal. A portion is shown here for guidance regarding its form and content.

and SN 2003XX, which resided less than 0."05 from the sharp nucleus of bright elliptical hosts and for which the technique of matching PSFs was used (Alard \& Lupton 1998).

For NICMOS imaging, the necessity of obtaining "clean" subtraction templates was judged for each object based on the complexity of the SN site in the bluer, F850LP templates. We judged that late-time templates were needed, and these were obtained for SN 2002hp, SN 2002ki, and SN 2003az. As with the ACS data, only SN 2002hp necessitated image convolution to match the PSF before subtraction.

The magnitudes of the SNe in the ACS images were calculated by fitting a PSF produced using bright comparison stars scaled to match the infinite-aperture zero points of M. Sirianni et al. (2004, in preparation). All ACS passband magnitudes are given as Vega-normalized magnitudes. Residual "sky" flux was measured using annuli centered on the $\mathrm{SN}$ and with an inner and outer radius of 0 "'66 and 1."00, respectively. The center of the PSF fit was determined from a centroid of a stack of all $\mathrm{SN}$ images.

Gilliland \& Riess (2002) have shown that the encircled energy of red stars in the IR is more dispersed than for blue stars, likely because of backside scattering from the ACS WFC CCD mounting. This is an important effect for images in F850LP with SNe Ia at $z>0.8$, which are considerably red $(i-z \approx 1 \mathrm{mag})$ and can be expected to suffer the "red halo" effect. To measure the SNe Ia in these images, we used a few bright, red $(i-z=1.0 \mathrm{mag})$ field stars as a PSF template.

Statistical uncertainties for the SN magnitudes were determined by adding and recovering artificial PSFs with the measured SN flux (Schmidt et al. 1998). Additional uncertainty was included for the shot noise of the measured SN flux. We found excellent agreement between the uncertainties calculated empirically and synthetically from the STScI exposure-time calculator.

NICMOS measurements of supernovae were similar, except that our model PSF and zero points were calculated from observations of standard stars P330E and G191B2B in Cycle 11 (M. Dickinson et al. 2003, private communication). For SN 2003es and SN 2003ak, which have noncomplex backgrounds, the underlying "sky" was determined from isophotal modeling of the host. For SN 2003dy and SN 2003eq the mean background was estimated with local apertures. Table 2 includes the measured magnitudes of the $\mathrm{SNe}$ Ia presented here. Figure 2 shows their light curves.

\subsection{Spectra}

Supernovae are best classified by the presence and absence of diagnostic features in their spectra (see Filippenko 1997 for review). For 14 of the $17 \mathrm{SNe}$ listed in Table 1, we obtained spectra of the $\mathrm{SNe}$ near maximum light. For two of these (SN 2003XX and SN 2002hp), bright, elliptical hosts yielded redshifts but overwhelmed the spectra of the SNe. For the remaining three (SN 2003ak, SN 2003aj, and SN 2002fw), spectra of the hosts to identify their redshifts were obtained when the $\mathrm{SNe}$ were no longer visible. Spectra of the 12 visible $\mathrm{SNe}$ are shown in Figure 3, and details of all the spectroscopy are provided in Table 3 .

To classify the SNe, the detected SN spectra (shown in Fig. 3) were cross-correlated with template spectra (after removal of the continuum) to identify their type and redshift using the SNID algorithm (Tonry et al. 2003). For the cases listed in Table 3 for which narrow-line host emission was identified, the redshift was constrained to the value determined from the host emission before cross-correlation. For cases where the spectra were of low $\mathrm{S} / \mathrm{N}$, the significance of an SN Ia classification is much greater if the SN redshift is fixed a priori by host emission. For all 12 spectra shown in Figure 3, SNID provided a significant classification for each as Type Ia. Although the diagnostic used by the SNID algorithm relies on the whole spectrum, the majority of these $\mathrm{SNe}$ can also be classified as Type Ia from the presence of Si II absorption at $4130 \AA$ (Coil et al. 2000). Specifically, $\mathrm{Si}$ II absorption is detected in the two highest redshift spectra presented here, SN 2003dy and SN 2002fw. Broad Ca II absorption near $3750 \AA$ is visible in all the spectra as well, but this feature is much less secure than $\mathrm{Si}$ II for $\mathrm{SN}$ Ia classification.

For two SNe (SN 2003XX and SN 2002hp) whose spectra were dominated by those of their hosts, the nature of their red, elliptical hosts allowed us to classify them as highly probable SNe Ia (as was previously the case with SN $1997 \mathrm{ff}$; Riess et al. 2001).

For the three SNe without any spectroscopy but with host redshifts, classification requires greater consideration. Based on the UV color selection method described by Riess et al. (2004), SN 2002fx, SN 2003ak, and SN 2003aj are likely to be SNe Ia because of their red discovery far-UV colors. However, further follow-up of SN 2003aj yielded a rapid decline, uncharacteristic of normal SNe Ia (see L.-G. Strolger et al. 2004, in preparation). The photometric records of the other two are consistent with SNe Ia. A prediscovery observation of SN 2003ak on the rise is also consistent with the narrow range of the rise behavior of $\mathrm{SNe}$ Ia, which rise in 3 weeks (Riess et al. 1999b), but not with the majority of core-collapse $\mathrm{SNe}$, which typically rise in 2 weeks or less, indicating that it is very likely to be an SN Ia. 

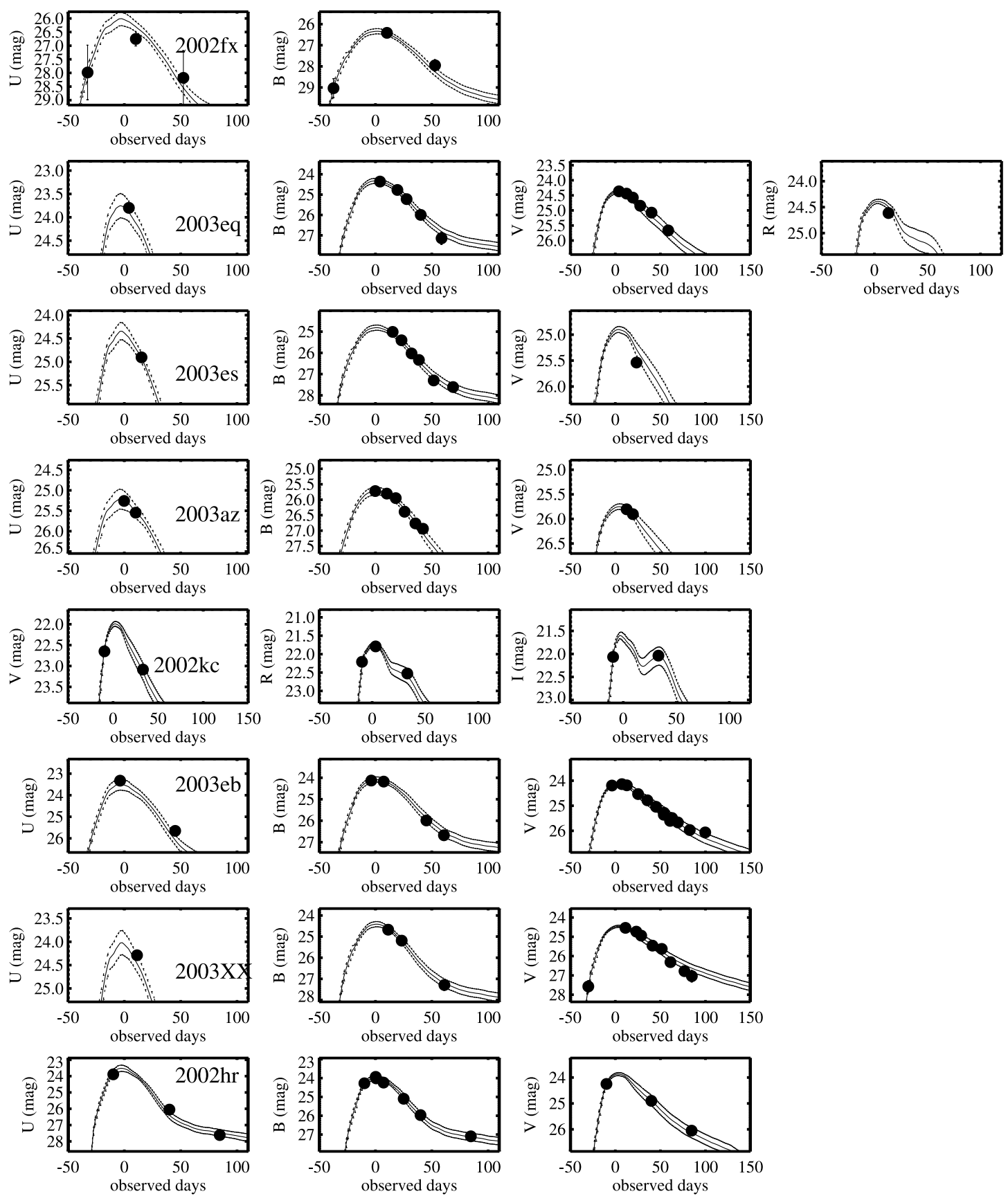

FIG. 2.-Multicolor light curves of SNe Ia. For each SN Ia, multicolor photometry transferred to rest-frame passbands is plotted. The individual, best-fit MLCS2k2 model is shown as a solid line, with a $\pm 1 \sigma$ model uncertainty, derived from the model covariance matrix, above and below the best fit.

For the 16 SNe Ia (excluding SN 2003aj), we have a sufficient quality of photometry to yield robust luminosity distances.

\section{LIGHT-CURVE FITTING}

Distances to $\mathrm{SNe}$ Ia with individual precision approaching $7 \%$ can be measured utilizing empirical relationships between light-curve shape and luminosity, as well as color-curve shape and extinction. Our primary analysis uses a revision of the multicolor light-curve shape (MLCS) fitting method (Riess et al. 1995, 1996a, 1998) described by Jha (2002) and S. Jha et al. (2004a, in preparation) and hereafter referred to as "MLCS2k2." Previous versions of MLCS fit rest-frame BVRI, but MLCS2k2 includes $U$-band templates based on a new set of 25 well-observed SNe Ia in the $U$ band from Jha (2002) and
S. Jha et al. (2004b, in preparation). This UV extension of MLCS allows us to extend the Hubble diagram of SNe Ia to $z>1$ using light curves observed in the reddest available band on ACS. The results from Jha (2002) demonstrate that rest-frame $U$-band light curves provide similar information on the epoch of maximum, relative luminosity, reddening, and distance as optical light curves, albeit with lower precision (by a factor of 1.5). Because the MLCS2k2 covariance matrix contains the bandpass-dependent variance of SNe Ia (primarily in the form of autocorrelation along the diagonals, as well as in the form of two-point covariance in off-diagonal terms), the $U$-band light curves can be used together with optical lightcurve data with the appropriate weight and propagation of uncertainty in the multicolor fit. 

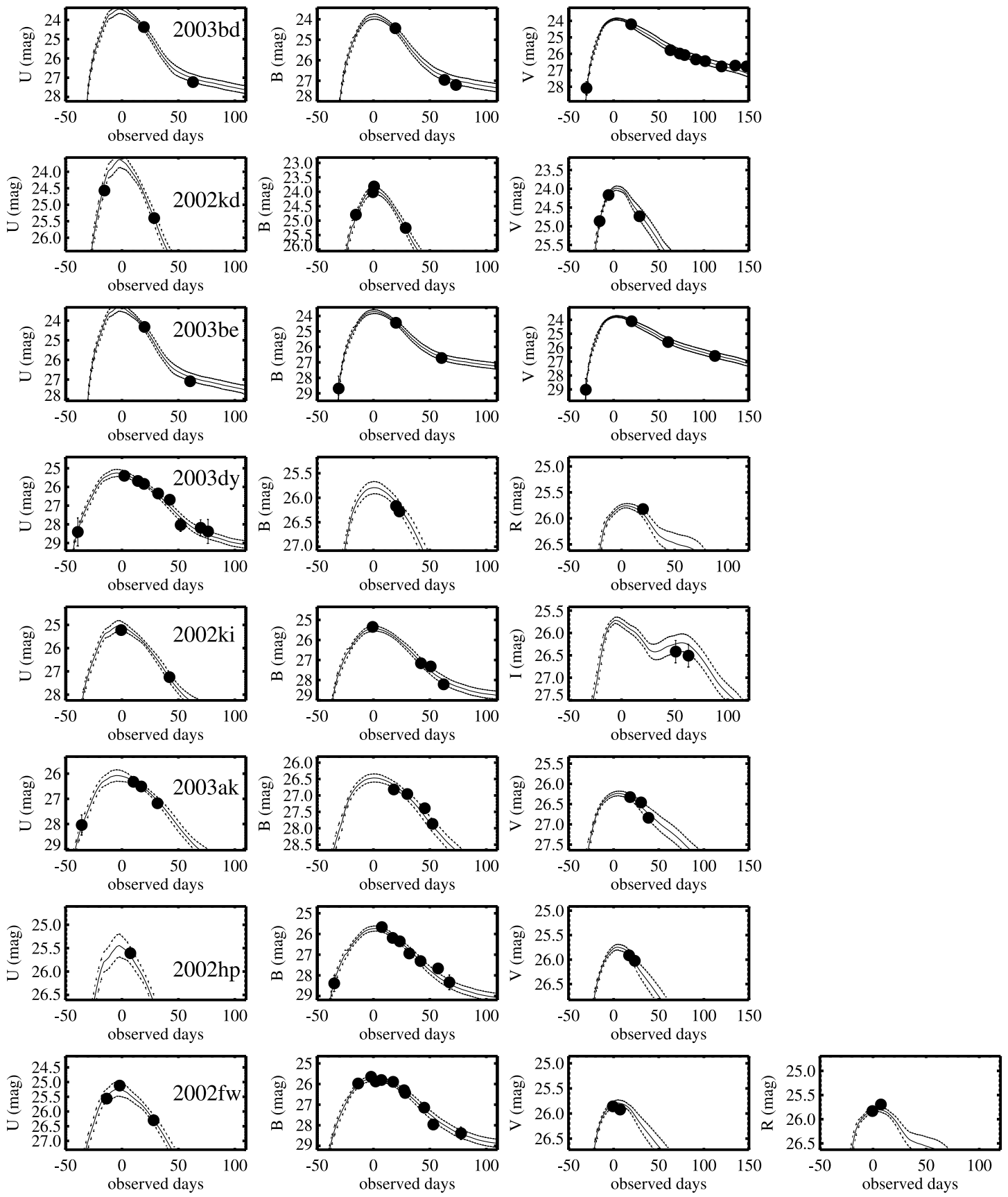

FIG. 2.-Continued

Additional improvements to MLCS2k2 include a more self-consistent treatment of the allowed range of extinction and extinction laws, as well as an improved determination of the unreddened SN Ia color (see S. Jha et al. 2004a, in preparation, for details). This method still empirically models a light curve as the sum of a fiducial template and a set of phase-dependent vectors (linear and quadratic) whose contribution scales with the luminosity offset from the fiducial curve at peak. Luminosity corrections are not extrapolated beyond the range observed in the local sample. Simultaneous fitting in multiple colors constrains the line-of-sight reddening and distance.

Extinction priors are used together with the observed reddening to estimate the expected dimming of the SN magnitudes resulting from dust. Recent analyses by the high- $z$ supernova search team (HZT; Schmidt et al. 1998) and the supernova cosmology project (SCP; Perlmutter et al. 1997), published respectively by Tonry et al. (2003) and Knop et al. (2003), make use of a Galactic prior for the extinction law (which is corroborated at low redshifts; Riess et al. 1996b) and flag SNe with large reddening to reduce systematic errors from nonGalactic type dust. The HZT has employed an exponential extinction prior whose functional form derives from modeled lines of sight (Hatano et al. 1998) and from the a posteriori distribution of extinction values. Here we utilize the exponential prior on extinction and the Gaussian prior on the intrinsic color derived from the a posteriori distribution of S. Jha et al. (2004a, in preparation) and flag SNe with large measured reddening as unreliable. Each SN Ia is corrected for Galactic reddening as estimated in the direction of each SN by Schlegel 

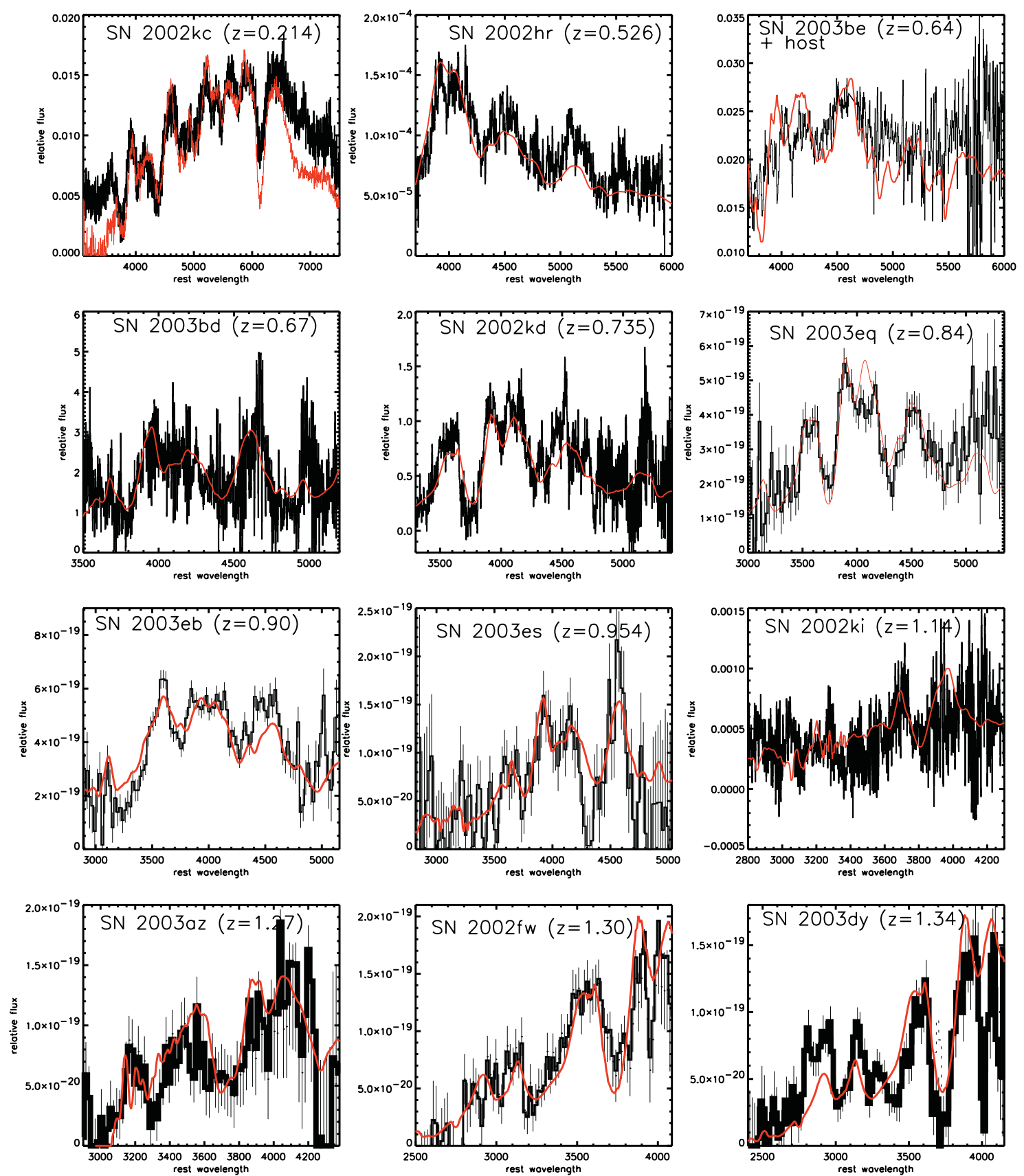

FIG. 3.-Identification spectra (in $f_{\lambda}$ ) of 12 of the new HST-discovered high-redshift SNe Ia, shown in the rest frame. Classification features are analyzed in $\S 2.3$. The data are compared to nearby SN Ia spectra of the same age as determined by the light curves (see Table 3). Classification of the five SNe without spectra (SN 2003XX, $z=0.94$; SN 2002fx, $z=1.40$; SN 2002hp, $z=1.31$; SN 2003ak, $z=1.55$; and SN 2003aj, $z=1.31$ ) are discussed in $\S \S 2.3$ and 3.1 .

et al. (1998) before the observed colors are used to estimate the host reddening.

$K$-corrections are used to account for the SN redshift and provide a transformation between an observed-frame magnitude and a rest-frame magnitude (Oke \& Sandage 1968). We use composite spectra of SNe Ia from Nugent et al. (2002) to calculate Vega-normalized "cross-band" $K$-corrections. Individual $K$-corrections were calculated to the best matching passbands for the appropriate phase and redshift of the SN. Each $\mathrm{SN}$ is then fitted using MLCS2k2 to provide a custom model of the phase-dependent colors. The colors of the Nugent et al. (2002) spectral energy distributions are then matched to the model by multiplication of a spline interpolation. Next, the $K$-corrections are recalculated, and the process continues until convergence (usually after $2-3$ iterations). The final $K$-corrections are given for each measured magnitude in Table 2. The MLCS2k2 fits to each of the 16 new SNe Ia are shown in Figure 2, and the fit parameters are given in Table 4.

We have also used an additional light-curve fitting method, the "Bayesian Template Method" (BATM; Tonry et al. 2003), to provide independent estimates of the luminosity distances of the new SN Ia data presented here. BATM is a "templatefitting" method that seeks to identify close matches between an individual SN Ia and a well-observed, local counterpart and then calculates their distance ratio by synthetically redshifting the nearby template to the observed frame. The current realization of BATM has far fewer $U$-band examples than MLCS2k2 because BATM was developed without the 
TABLE 3

Spectroscopic Data

\begin{tabular}{|c|c|c|c|c|}
\hline SN & UT Date & Instrument & $\begin{array}{l}\text { Exposure } \\
\text { (s) }\end{array}$ & $z$ \\
\hline $2002 \mathrm{fw}$ & 2002 Sep 31 & HST ACS & 15000 & $1.30^{\mathrm{a}, \mathrm{b}}$ \\
\hline 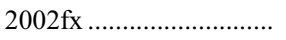 & 2003 Sep 14 & Keck II NIRSPEC & 2000 & $1.40^{\mathrm{c}, \mathrm{d}}$ \\
\hline \multirow[t]{2}{*}{ 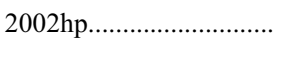 } & 2002 Nov 7 & Keck I LRIS & 7800 & $1.305^{\mathrm{c}, \mathrm{e}}$ \\
\hline & $2002 \operatorname{Nov} 7$ & VLT FORS & 14000 & $1.305^{\mathrm{c}, \mathrm{e}}$ \\
\hline 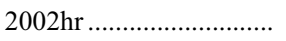 & 2002 Nov 8 & Keck I LRIS & 7800 & $0.526^{\mathrm{b}, \mathrm{f}}$ \\
\hline 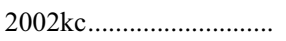 & $2003 \operatorname{Jan} 7$ & Keck I LRIS & 1500 & $0.216^{\mathrm{b}, \mathrm{f}}$ \\
\hline 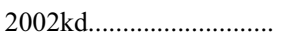 & 2003 Jan 1 & Magellan LDSS & 7200 & $0.735^{\mathrm{b}, \mathrm{f}}$ \\
\hline 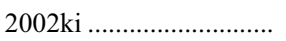 & 2003 Jan 7 & Keck I LRIS & 2700 & $1.141^{\mathrm{b}, \mathrm{f}}$ \\
\hline 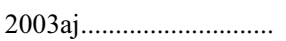 & 2003 Oct $1-3$ & VLT FORS2 & 16800 & $1.307^{\mathrm{c}, \mathrm{g}}$ \\
\hline 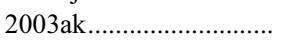 & 2003 Sep 11 & Keck II NIRSPEC, VLT FORS2 & 14000 & $1.551^{\mathrm{c}, \mathrm{d}}$ \\
\hline 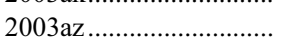 & 2003 Mar 3 & HST ACS & 6500 & $1.27^{\mathrm{a}, \mathrm{b}}$ \\
\hline 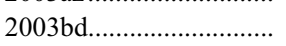 & 2003 Feb $27 / 28$ & Keck I LRIS & 16500 & $0.67^{\mathrm{a}, \mathrm{b}}$ \\
\hline 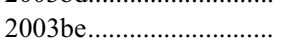 & 2003 Feb 28 & Keck I LRIS & 5400 & $0.64^{\mathrm{b}, \mathrm{f}}$ \\
\hline 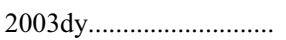 & 2003 Apr 16 & HST ACS & 15000 & $1.34^{\mathrm{b}, \mathrm{h}}$ \\
\hline 2003XX & 2003 Apr 16 & HST ACS & 15000 & $0.935^{\mathrm{e}, \mathrm{h}}$ \\
\hline $2003 \mathrm{eb}$ & 2003 Apr 16 & HST ACS & 15000 & $0.899^{\mathrm{b}, \mathrm{h}}$ \\
\hline 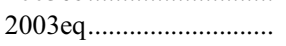 & 2003 Jun 2 & HST ACS & 6000 & $0.839^{\mathrm{a}, \mathrm{b}}$ \\
\hline 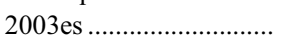 & 2003 Jun 2 & HST ACS & 6000 & $0.954^{\mathrm{b}, \mathrm{h}}$ \\
\hline
\end{tabular}

${ }^{\text {a }}$ From cross-correlation with broad SN features.

b Classified as SN Ia with high confidence from spectrum.

${ }^{c}$ From narrow features in the host-galaxy spectrum.

${ }^{\mathrm{d}}$ Photometric properties indicate likely SN Ia.

${ }^{\mathrm{e}}$ Classified as SN Ia with high confidence from early-type, red host.

${ }^{\mathrm{f}}$ From both cross-correlation with broad SN features and narrow features in the host-galaxy spectrum.

${ }^{g}$ Uncertain type.

${ }^{\mathrm{h}}$ From cross-correlation with broad SN features, Cowie et al. (2004), and Wirth et al. (2004).

Jha (2002) data. Consequently, BATM did not converge on a solution for three sparsely observed $\mathrm{SNe} \mathrm{Ia}(\mathrm{SNe} 2002 \mathrm{kc}$, 2003be, and 2002fx) whose MLCS2k2 fits relied on sampling in the rest-frame $U$ band.

\subsection{The Ground-based Discovery Set}

We construct the expansion history of the universe by using this new set of HST-discovered objects, together with published observations of supernovae over a wide range in redshift. Tonry et al. (2003) have recently compiled the distances and redshifts for $172 \mathrm{SNe}$ Ia. Using available results from different light-curve fitting methods (including BATM, MLCS, $\Delta m_{15}$, snapshot, and stretch) for each SN Ia, Tonry et al. (2003) corrected for zero-point differences between methods and provided best estimates of the distance to each SN Ia from a median of the distance estimates from individual methods.

Although the Tonry et al. (2003) data set represented the state of the art in 2003 February, when it was submitted, there have been some significant developments since then that need to be included to build the most reliable data set for analyzing the HST-discovered objects. For the SCP, Knop et al. (2003) report on a new set of $11 \mathrm{SNe}$ Ia at $0.4<z<0.85$, as well as on a reanalysis of the original high-redshift SNe Ia from the SCP (Perlmutter et al. 1999). In this reanalysis they now exclude 15 of the 42 high-redshift SNe from Perlmutter et al. (1999) because of inaccurate color measurements and uncertain classification. Knop et al. (2003) flag an additional 6 of the original $42 \mathrm{SNe}$, as well as 5 of the new $11 \mathrm{SNe}$, as likely SNe Ia but failing a "strict SN Ia" sample cut. For the HZT, Barris et al. (2004) report on a large set of new high-redshift $\mathrm{SNe}$ (22 in all), with widely varying degrees of completeness of the spectroscopic and photometric records. Finally, Blakeslee et al. (2003) report on two new SNe Ia discovered with ACS on HST. The development of the MLCS2k 2 method, which includes $U$-band observations when they are available, makes it worthwhile to revisit the previously published data.

We recompiled a set of previously observed SNe Ia, relying on large, published samples whenever possible to reduce systematic errors from differences in calibration. To compile SNe Ia at $0.01<z<0.15$ we used the three largest, modern data sets of such SNe Ia published to date: the Calán-Tololo Survey (29 SNe Ia; Hamuy et al. 1996a, 1996b), the CfA

TABLE 4

$\chi^{2}$ Comparison of Gold Set Data to Models

\begin{tabular}{|c|c|}
\hline Model & $\chi^{2}$ (for $\left.157 \mathrm{SNe} \mathrm{Ia}\right)$ \\
\hline$\Omega_{M}=0.27, \Omega_{\Lambda}=0.73$ & 178 \\
\hline$\Omega_{M}=1.00, \Omega_{\Lambda}=0.00$ & 325 \\
\hline$\Omega_{M}=0.00, \Omega_{\Lambda}=0.00 \ldots \ldots$ & 192 \\
\hline High-redshift gray dust (with $\Omega_{M}=1.00, \Omega_{\Lambda}=0.00$ ) ..................... & 307 \\
\hline 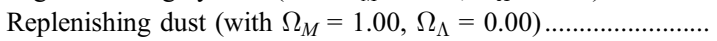 & 175 \\
\hline 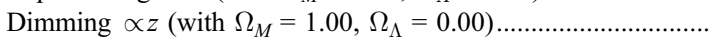 & 253 \\
\hline
\end{tabular}


Survey I (22 SNe Ia; Riess et al. 1999a), and the CfA Survey II (44 SNe Ia; S. Jha et al. 2004b, in preparation). ${ }^{10}$ At higher redshifts we used SN Ia photometry published by Riess et al. (1998, 2001), Tonry et al. (2003), Knop et al. (2003), and Barris et al. (2004), as well as SNe Ia tabulated by Tonry et al. (2003) from N. Suntzeff et al. (2004, in preparation), B. Leibundgut et al. (2004, in preparation), A. Clochiatti et al. (2004, in preparation), and S. Jha et al. (2004c, in preparation). Despite the apparently large number of sources of data, the majority of the data at $z>0.1$ comes from the HZT (Schmidt et al. 1998; Riess et al. 1998), and thus the methods used to calibrate all of these SNe Ia are extremely similar (and familiar to the authors of the present paper).

In order to reduce systematic errors that arise from differences in light-curve fitting (and $K$-correcting) methods, we have made every effort to consistently refit all the past data with a single method, MLCS2k2 (S. Jha et al. 2004a, in preparation). An exception was made for the high-redshift SNe Ia from the SCP (Perlmutter et al. 1999) because photometry of these $\mathrm{SNe}$ Ia remains unpublished. For these we utilized the reanalyzed distances as given by Knop et al. (2003). We transformed them to the MLCS2k2 distance scale by solving for the SN Ia luminosity zero point required to match the mean distances to low-redshift objects fitted by the two methods.

Unfortunately, a large variation exists in the quality and breadth of the photometric and spectroscopic records of individual SNe. Ideally, each SN Ia would have the same well-defined spectroscopic features (Filippenko 1997), a spectroscopic redshift, and well-sampled light curves and color curves. However, this is often not the case. As with the photometry, most of the extant high-redshift spectra from the SCP have not been published (e.g., Perlmutter et al. 1999; Knop et al. 2003). This makes it difficult to apply a uniform set of criteria to $\mathrm{SNe}$ Ia in constructing a cosmological sample.

To reflect the differences in the quality of the spectroscopic and photometric record for individual supernovae, we divide the objects into "high-confidence" (hereafter "gold") and "likely but not certain" (hereafter "silver") subsets. Ideally, we would assign each supernova a weight in any overall fit that reflected its individual uncertainty. However, distance errors resulting from spurious problems more common to lower confidence SNe Ia, such as SN misclassification, large extinction (amplifying uncertain extragalactic extinction laws), and poorly constrained colors, are difficult to quantify. So we use the coarser approach of separating the high-confidence gold events from the larger set.

We adopted in our gold set all SNe Ia that were included by the original sources in their most stringent subsets (when such discriminations were made). Any SN Ia flagged as having a cause for a specific concern was not included in this set. The two primary reasons for rejecting an SN Ia from this set were that (1) the classification, although plausible, was not compelling (see discussion) and (2) the photometric record was too incomplete to yield a robust distance (i.e., the number of model parameters is roughly equal to the number of effective samplings of the light curve).

$\mathrm{SNe}$ Ia included in previous cosmological samples but rejected from our gold sample include SN 1999fh (poorly constrained light curve; Tonry et al. 2003), SN 1997ck (poor color information; Garnavich et al. 1998), all "snapshot" $\mathrm{SNe}$

\footnotetext{
${ }^{10}$ Note that the CfA SNe were generally discovered by other searches, especially the Lick Observatory SN Search with the Katzman Automatic Imaging Telescope (Filippenko et al. 2001; Filippenko 2002).
}

Ia from Riess et al. (1998), 15 SNe Ia from Perlmutter et al. (1999) later discarded by Knop et al. (2003) as well as 11 additional SNe flagged by Knop et al. (2003; flag values 1, 2, $3)$, SNe Ia from Barris et al. (2004) without SNID classification, and any SN Ia with more than 1 mag of extinction or whose light curve begins more than 10 rest-frame days after maximum as determined from the MLCS2k2 fit.

The same criteria were applied to the GOODS SNe Ia, whose individual classifications were described in $\S 2.3$. As a result, two of these $\mathrm{SNe}$ were rejected from the gold sample: SN 2002fx, whose classification is not certain enough, and SN $2002 \mathrm{kc}$, whose fit indicates more than 1 mag extinction. The gold set contains a total of $157 \mathrm{SNe}$ Ia.

The silver set contains the objects identified by the above sources as likely $\mathrm{SNe}$ Ia but failing one criterion for inclusion in the gold category. The silver set includes a total of 29 $\mathrm{SNe}$ Ia. SNe failing more than one criterion were excluded from the analyses. The final membership rosters of the subsets are tabulated in the Appendix.

For most of our cosmological analyses we focus on results derived from the gold set, but for a few analyses with the largest number of free parameters (and thus the most limited in statistical inference), we include the silver set (with the caveats arising from its reduced reliability).

\section{COSMOLOGICAL CONSTRAINTS}

Distance estimates from SN Ia light curves are derived from the luminosity distance

$$
d_{L}=\left(\frac{\mathcal{L}}{4 \pi \mathcal{F}}\right)^{1 / 2}
$$

where $\mathcal{L}$ and $\mathcal{F}$ are the intrinsic luminosity and observed flux of the SN within a given passband, respectively. Equivalently, logarithmic measures of the flux (apparent magnitude, $m$ ) and luminosity (absolute magnitude, $M$ ) were used to derive extinction-corrected distance moduli, $\mu_{0}=m-M=$ $5 \log d_{L}+25$ ( $d_{L}$ in units of megaparsecs). In this context, the luminosity is a "nuisance parameter" whose value is unimportant for kinematic (and most cosmological) studies. We have used the MLCS2k2 method and the data described in $\S 2$ to derive accurate and individual relative distance moduli for the sets of $\mathrm{SNe}$ described in $\S 3$.

In Figure 4 we show the Hubble diagram of distance moduli and redshifts for the new HST-discovered SNe Ia in the gold and silver sets. Although these new SNe Ia span a wide range of redshift $(0.21<z<1.55)$, their most valuable contribution to the SN Ia Hubble diagram is in the highest redshift region, where they effectively delineate the range at $0.85<z<1.55$ with 11 new SNe Ia, including 6 of the 7 highest redshift SNe known (the seventh being SN 1997ff; Riess et al. 2001).

The relationship between distance and redshift over a significant fraction of the Hubble time can be considered either empirically as a record of the (integrated) expansion history of the universe or theoretically as constraints on the mass-energy terms contained in the Friedman equation and affecting the expansion. In the next subsections we consider both approaches.

\subsection{Expansion History: A Kinematic Description}

It is valuable to consider the distance-redshift relation of $\mathrm{SNe}$ Ia as a purely kinematic record of the expansion history of the universe, without regard to its cause. An empirical description of the time variation of the scale factor $a(t)$ can provide answers to basic questions (e.g., "When was the universe [if 


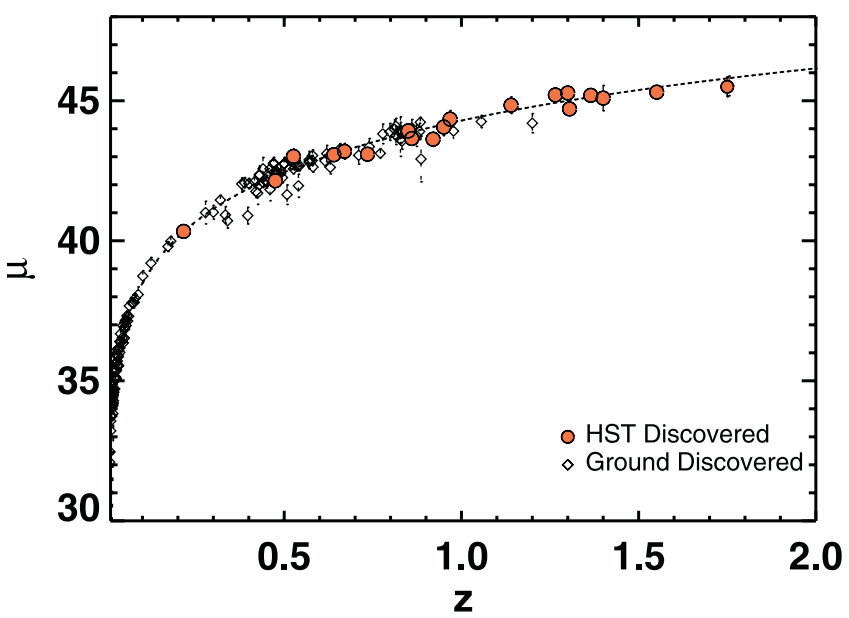

FIG. 4.-MLCS2k2 SN Ia Hubble diagram. SNe Ia from ground-based discoveries in the gold sample are shown as diamonds; HST-discovered SNe Ia are shown as filled symbols. Overplotted is the best fit for a flat cosmology: $\Omega_{M}=0.29, \Omega_{\Lambda}=0.71$.

ever] accelerating or decelerating?") and model-independent constraints with which to test cosmological models.

Following Turner \& Riess (2002), we empirically define the luminosity distance in Euclidean space (i.e., $\Omega_{\text {total }}=1.0$; as motivated by inflation) as the integral of the inverse of the preceding expansion rate,

$$
\begin{aligned}
d_{L} & =c(1+z) \int_{0}^{z} \frac{d u}{H(u)} \\
& =c(1+z) H_{0}^{-1} \int_{0}^{z} \exp \left\{-\int_{0}^{u}[1+q(u)] d \ln (1+u)\right\} d u,
\end{aligned}
$$

where

$$
\begin{gathered}
H(z)=\frac{\dot{a}}{a} \\
q(z) \equiv \frac{-\ddot{a} / a}{H^{2}(z)}=\frac{d H^{-1}(z)}{d t}-1 .
\end{gathered}
$$

Note that equation (2) is not an approximation but is an exact expression for the luminosity distance in a geometrically flat universe (although generalizable for nonzero curvature), given an expression for the epoch-dependent deceleration parameter $q(z)$ and the present Hubble constant $H_{0}$. Here we employ equation (2) as a kinematic model of the SN Ia data with parametric representations for $q(z)$.

Given evidence that the universe has recently been accelerating [i.e., $q(z \sim 0)<0$ ], hints that it may have once been decelerating [i.e., $q(z>1)>0$; Riess et al. 2001; Turner \& Riess 2002], and the large leverage in redshift of the current SN sample, we consider resolving $q(z)$ into two distinct components or epochs. A linear two-parameter expansion for $q(z)$ that is continuous and smooth is $q(z)=q_{0}+z d q / d z$, where $d q / d z$ is defined to be evaluated at $z=0$.

The likelihood for the parameters $q_{0}$ and $d q / d z$ can be determined from a $\chi^{2}$ statistic, where

$$
\chi^{2}\left(H_{0}, q_{0}, \frac{d q}{d z}\right)=\sum_{i} \frac{\left[\mu_{p, i}\left(z_{i} ; H_{0}, q_{0}, d q / d z\right)-\mu_{0, i}\right]^{2}}{\sigma_{\mu_{0, i}}^{2}+\sigma_{v}^{2}}
$$

$\sigma_{v}$ is the dispersion in supernova redshift (transformed to units of distance moduli) due to peculiar velocities, and $\sigma_{\mu_{0, i}}$ is the uncertainty in the individual distance moduli. This term also includes the uncertainty in galaxy redshift. Because of the extreme redshift of our distant sample and the abundance of objects in the nearby sample, our analysis is insensitive to the value that we assume for $\sigma_{v}$ within its likely range of $200 \mathrm{~km}$ $\mathrm{s}^{-1} \leq \sigma_{v} \leq 500 \mathrm{~km} \mathrm{~s}^{-1}$. For our analysis we adopt $\sigma_{v}=$ $400 \mathrm{~km} \mathrm{~s}^{-1}$. For high-redshift SNe Ia whose redshifts were determined from the broad features in the SN spectrum, we add $2500 \mathrm{~km} \mathrm{~s}^{-1}$ in quadrature to $\sigma_{v}$.

Marginalizing our likelihood functions over the nuisance parameter $H_{0}$ (by integrating the probability density $P \propto$ $e^{-\chi^{2} / 2}$ for all values of $H_{0}$ ) yields the confidence intervals shown in Figure 5. As shown, both the gold set and the gold and silver sets together strongly favor a universe with recent acceleration $\left(q_{0}<0\right)$ and previous deceleration $(d q / d z>0)$ with $99.2 \%$ and $99.8 \%$ likelihood (summed within this quadrant), respectively. With this same model we can also derive the likelihood function for the transition redshift $z_{t}$, defined as $q\left(z_{t}\right)=0$. Summing the probability density in the $q_{0}$ versus $d q / d z$ plane along lines of constant transition redshift, $z_{t}=$ $-q_{0} /(d q / d z)$, yields the likelihood function in Figure 5. We find a transition redshift of $z_{t}=0.46 \pm 0.13$. In Figure 6 we show the Hubble diagram for the $\mathrm{SNe}$ Ia compared to a discrete set of kinematic models.

An alternate, kinematic model is derived using the first three time derivatives of the scale factor. Following Visser (2003), the Hubble, deceleration, and jerk parameters are defined as

$$
\begin{gathered}
H(t)=+\dot{a} / a, \\
q(t)=-(\ddot{a} / a)(\dot{a} / a)^{-2}, \\
j(t)=+(\dot{\ddot{a}} / a)(\dot{a} / a)^{-3} .
\end{gathered}
$$

The deceleration and jerk parameters are dimensionless, and a Taylor expansion of the scale factor around $t_{0}$ provides

$$
\begin{aligned}
a(t)= & a_{0}\left\{1+H_{0}\left(t-t_{0}\right)-\frac{1}{2} q_{0} H_{0}^{2}\left(t-t_{0}\right)^{2}\right. \\
& \left.+\frac{1}{3 !} j_{0} H_{0}^{3}\left(t-t_{0}\right)^{3}+O\left[\left(t-t_{0}\right)^{4}\right]\right\},
\end{aligned}
$$

and hence for the luminosity distance (in Euclidean space),

$$
\begin{aligned}
d_{L}(z)= & \frac{c z}{H_{0}}\left[1+\frac{1}{2}\left(1-q_{0}\right) z\right. \\
& \left.-\frac{1}{6}\left(1-q_{0}-3 q_{0}^{2}+j_{0}\right) z^{2}+O\left(z^{3}\right)\right]
\end{aligned}
$$

(see Visser 2003).

Although related, the $j_{0}$ parameter as defined here and by Visser (2003) is not precisely equivalent to our previous $d q / d z$ parameter, providing an alternative parameterization. The SN subsets constrain the $j_{0}$ parameter to the positive domain at the $92 \%-95 \%$ confidence level. That is, the expansion history over the range of the $\mathrm{SN}$ data is equally well described by recent acceleration and a constant jerk. Models with discrete values of $j_{0}$ are shown in Figure 6. 

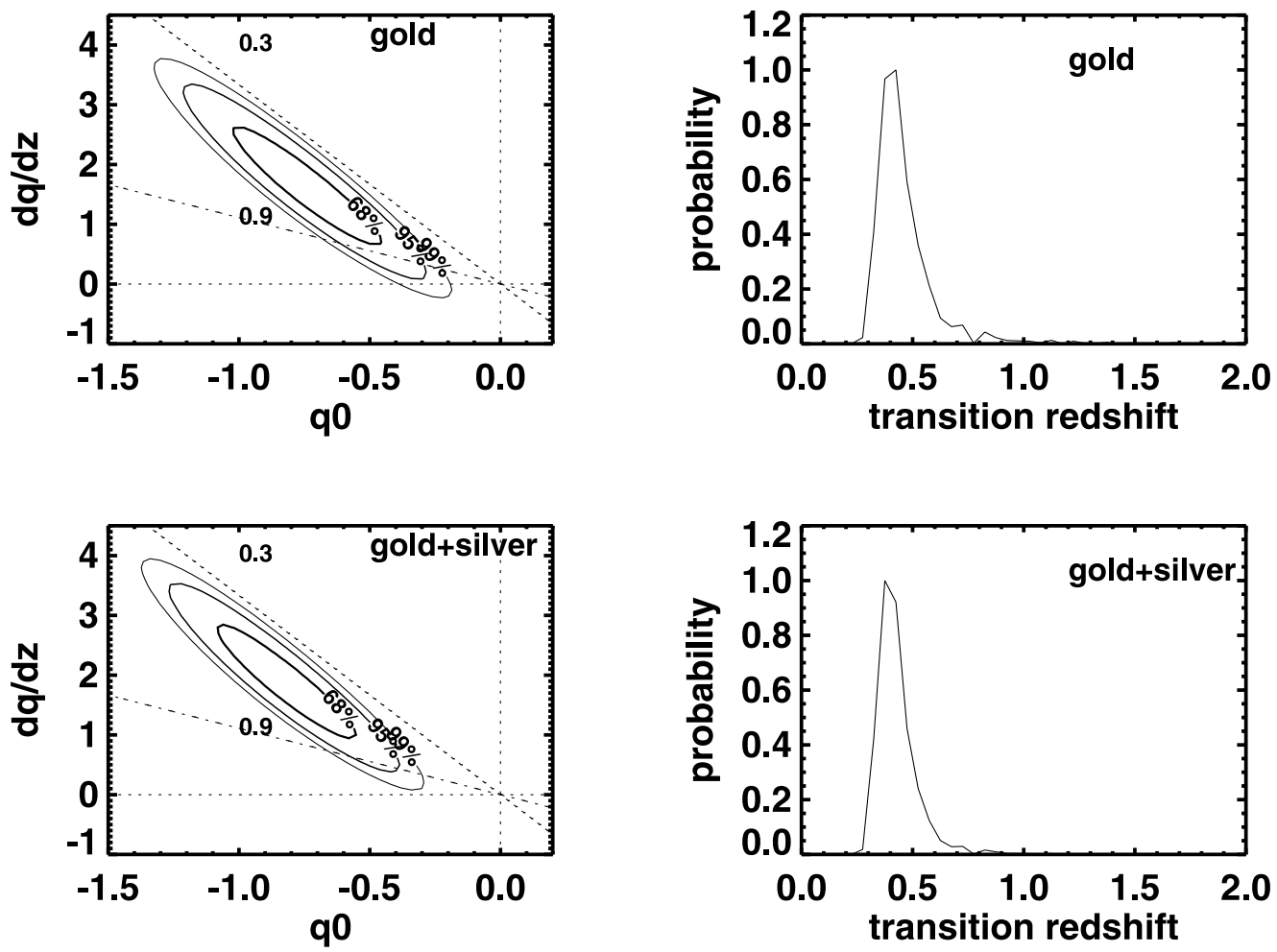

FIG. 5. - Left: Joint confidence intervals for a two-parameter model of the expansion history, $q(z)=q_{0}+z d q / d z$, from SNe Ia. The top left shows the constraints derived from the gold sample; the bottom left includes both gold and silver samples. For either set, the data favor the quadrant with recent acceleration $\left(q_{0}<0\right)$ and past deceleration $(d q / d z>0)$ with high confidence. Lines of fixed transition redshift $\left[q\left(z_{t}\right)=0\right]$ are shown. Right: Illustration of the likelihood function for the transition redshifts derived from the same samples.

In summary, we find strong evidence for a change in the sign of cosmic acceleration in the past.

\subsection{Cosmological Constant or Astrophysical Dimming?}

SNe Ia at $z \approx 0.5$ appear fainter by $\sim 0.25$ mag relative to a universe with $\Omega_{M}=0.3$ and $\Omega_{\Lambda}=0$, a result readily accom- modated by a cosmological constant with $\Omega_{\Lambda}=0.7$ (Riess et al. 1998; Perlmutter et al. 1999). Despite the lack of any independent evidence, an alternative explanation for this dimming could lie in the astrophysics of supernovae or in the propagation of their light to us. Speculative models for astrophysical contamination of the SN Ia signal have been

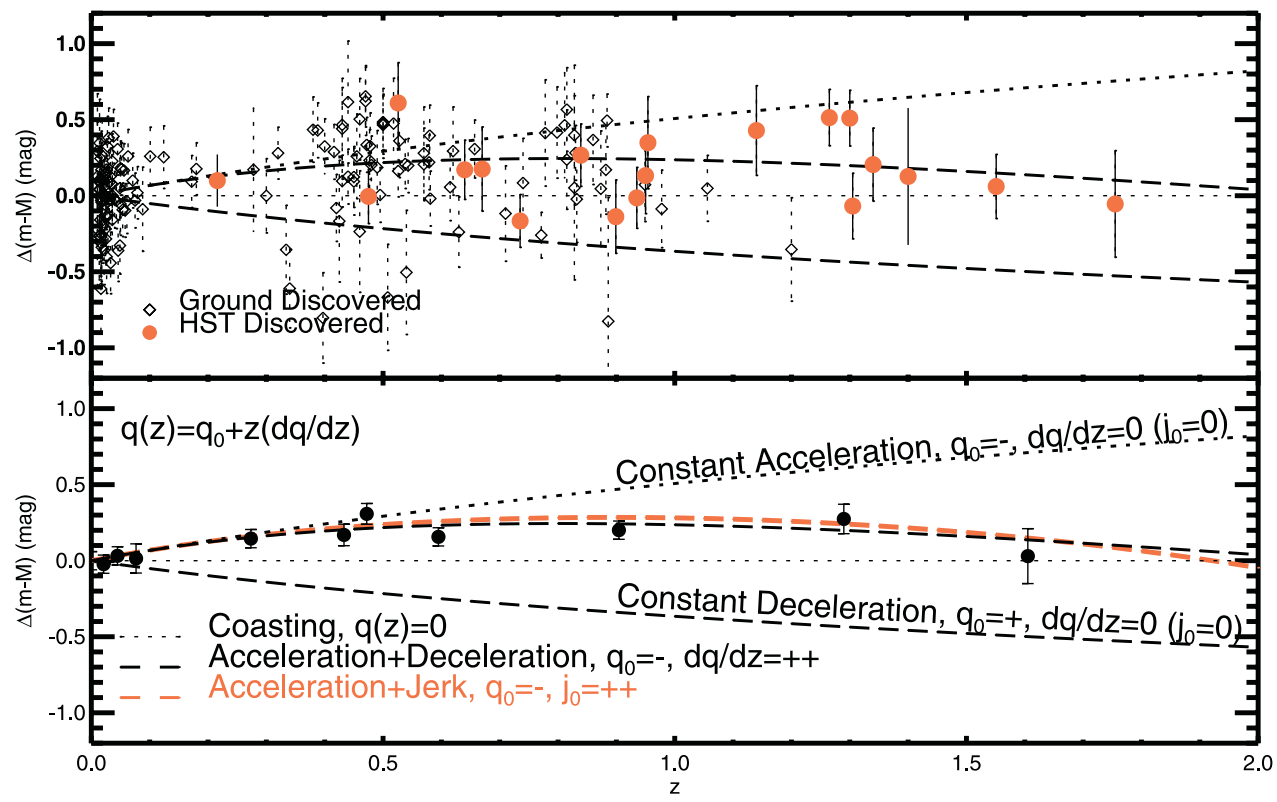

FIG. 6.-Kinematic SN Ia residual Hubble diagram. Top: SNe Ia from ground-based discoveries in the gold sample are shown as diamonds; HST-discovered SNe Ia are shown as filled symbols. Bottom: Weighted averages in fixed redshift bins are given for illustrative purposes only. Data and kinematic models of the expansion history are shown relative to an eternally coasting model, $q(z)=0$. Models representing specific kinematic scenarios (e.g., "constant acceleration") are illustrated. 


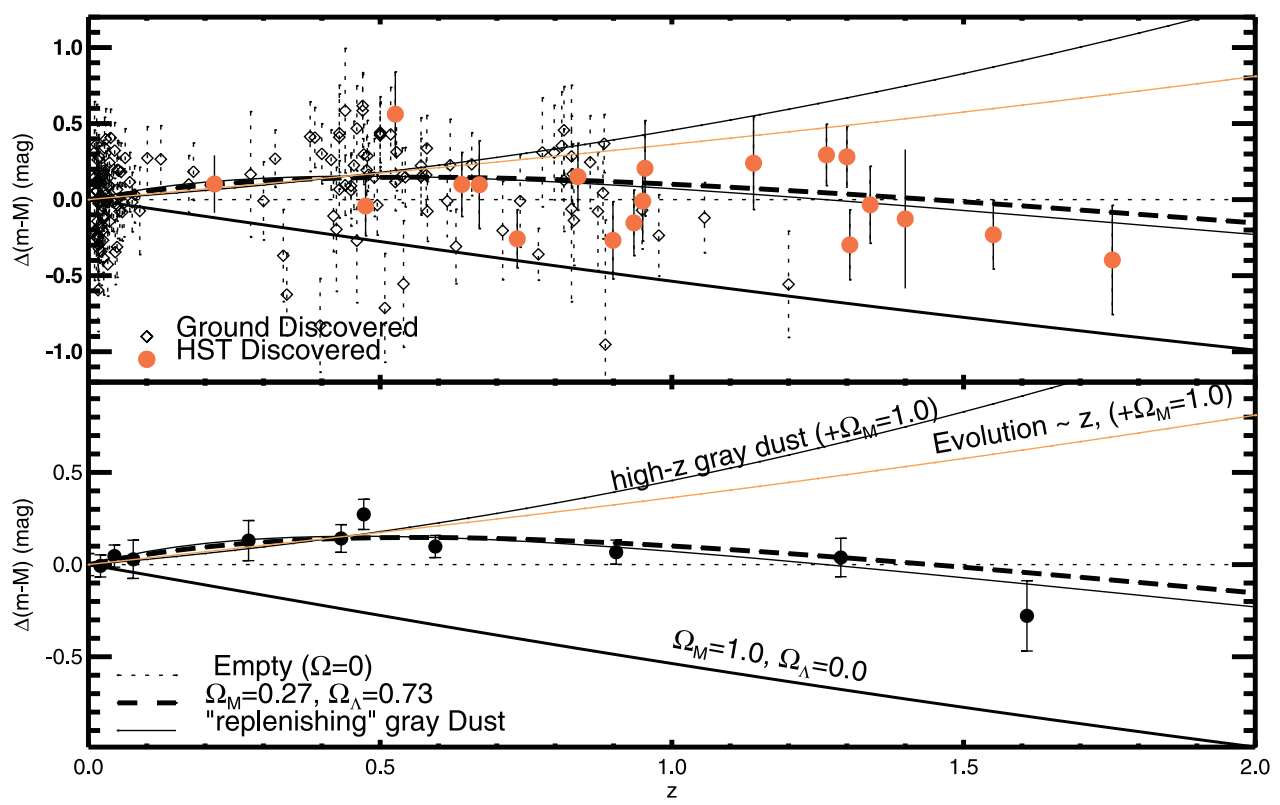

FIG. 7.-SN Ia residual Hubble diagram comparing cosmological models and models for astrophysical dimming. Top: SNe Ia from ground-based discoveries in the gold sample are shown as diamonds; HST-discovered SNe Ia are shown as filled symbols. Bottom: Weighted averages in fixed redshift bins are given for illustrative purposes only. Data and models are shown relative to an empty universe model $(\Omega=0)$. The $\chi^{2}$ fit statistics for each model are listed in Table 4 .

posited; these include extragalactic gray dust with negligible telltale reddening or added dispersion (Aguirre 1999a, 1999b; Rana 1979, 1980) and a pure luminosity evolution (Drell et al. 2000). Here we limit our consideration to the observable differences between these hypotheses.

The luminosity distance expected in a Friedmann-RobertsonWalker (FRW) cosmology with mass density $\Omega_{M}$ and vacuum energy density (i.e., the cosmological constant) $\Omega_{\Lambda}$ is

$$
\begin{aligned}
d_{L}= & c H_{0}^{-1}(1+z)\left|\Omega_{k}\right|^{-1 / 2} \operatorname{sinn}\left\{\left|\Omega_{k}\right|^{1 / 2}\right. \\
& \left.\times \int_{0}^{z} d z\left[(1+z)^{2}\left(1+\Omega_{M} z\right)-z(2+z) \Omega_{\Lambda}\right]^{-1 / 2}\right\},
\end{aligned}
$$

where $\Omega_{k}=1-\Omega_{M}-\Omega_{\Lambda}$, and "sinn" is $\sinh$ for $\Omega_{k}>0$ and $\sin$ for $\Omega_{k}<0$ (Carroll et al. 1992). For $\Omega_{k}=0$, equation (11) reduces to $\mathrm{cH}_{0}^{-1}(1+z)$ times the integral. With $d_{L}$ in units of megaparsecs, the predicted distance modulus is

$$
\mu_{p}=5 \log d_{L}+25
$$

Following Goobar et al. (2002) we consider two models of gray extinction by a homogeneous component of dust: $\rho_{\text {dust }}(z)=\rho_{\text {dust }}^{0}(1+z)^{\alpha}$, where

$$
\alpha(z)=\left\{\begin{array}{ll}
3 & \text { for all } z \quad(\text { high }-z \text { dust }) \\
0 & z>0.5(3 \text { for lower } z)
\end{array}\right. \text { (replenishing dust). }
$$

The "high-z dust" model represents a smooth background of dust present (presumably ejected from galaxies) at a redshift that is greater than that of the SN sample (i.e., $z>2$ ) and diluting as the universe expands. The total extinction is then calculated as the attenuation integrated along the photon path, $-2.5 \log \left\{\exp \left[\int_{0}^{z} \rho_{\text {dust }}(z) r(z) d z\right]\right\}$, where $r(z)$ is the coordinate distance traversed by the SN photons. A single free (opacity) parameter is fixed by requiring the total extinction at $z \approx 0.5$ to match the observed peak brightness of SNe Ia in a cosmology with $\Omega_{\Lambda}=0$.

The "replenishing dust" represents a constant density of dust that is continually replenished at precisely the same rate in which it is diluted by the expanding universe (i.e., $\alpha=0$ ). This model is also tuned to match the extinction implied by $\mathrm{SNe}$ Ia at $z \approx 0.5$ in the absence of a cosmological constant; hence it requires the tuning of two parameters (as well as fastmoving dust, which quickly provides a homogeneous background without added dispersion from uneven lines of sight). We also consider a third model (following Filippenko \& Riess 2001, Riess et al. 2001, and Blakeslee et al. 2003) to mimic simple evolution, which scales as $z$ in percent dimming. Our set of models for astrophysical dimming is not an exhaustive set of all possibilities but rather is drawn from physically motivated hypotheses (in contrast to purely parametric models of astrophysical dimming, e.g., Drell et al. 2000).

In Figure 7 we show the Hubble diagram of SNe Ia relative to the cosmological and astrophysical hypotheses. As seen in Table 4, the SN data set is consistent with an $\Omega_{M}=$ $0.27, \Omega_{\Lambda}=0.73$ cosmology, yielding $\chi^{2}=178$ for $157 \mathrm{SNe}$ Ia (degrees of freedom, dof; $\chi_{\text {dof }}^{2}=1.13$ ) in the gold set. The total $\chi^{2}$ is significantly worse for the high-redshift gray dust model $\left(\Delta \chi^{2}=122 ; 11 \sigma\right.$ for 1 dof $)$, as well as for the simple model of evolution with dimming $\propto z\left(\Delta \chi^{2}=70 ; 8 \sigma\right.$ for 1 dof), allowing us to reject both hypotheses with high confidence. Interestingly, the "replenishing dust" model is nearly indistinguishable from an $\Omega_{\Lambda}$ model because the dimming is directly proportional to distance traveled and thus mathematically quite similar to the effects of a cosmological constant. Consequently, we cannot discriminate this model from an $\Omega_{\Lambda}$-dominated model strictly from its behavior in the magnitude-redshift plane (and probably never will be able to, given the small magnitude differences). However, the finetuning required of this dust's opacity, replenishing rate, and velocity $\left(>1000 \mathrm{~km} \mathrm{~s}^{-1}\right.$ for it to fill space uniformly without adding detectable dispersion) makes it unattractive as a simpler alternative to a cosmological constant. 


\subsection{Exploring Dark Energy}

Despite the results of the last section that favor the dark energy interpretation of $\mathrm{SNe}$ Ia, we avoid using this conclusion as a starting point for exploring the nature of dark energy. To do so would be to engage in circular reasoning or to incur more free parameters than our limited data set can usefully constrain. Instead, we embark on a parallel study from the previous section. Here we use distance-independent information to justify the cosmological interpretation of SNe Ia and combine with other experiments to study dark energy.

The potential for luminosity evolution of corrected SN Ia distances has been studied using a wide range of local host environments. No dependence of the distance measures on the host morphology, mean stellar age, radial distance from the center, dust content, or mean metallicity has been seen (Riess et al. 1998; Perlmutter et al. 1999; Hamuy et al. 2000). No differences in the inferred cosmology were seen by Sullivan et al. (2003) for SNe Ia in early-type hosts or latetype hosts at high redshifts. These studies limit morphology dependence of SN Ia distances to the 5\% level. Detailed studies of distance-independent observables of SNe Ia, such as their spectral energy distribution and temporal progression, have also been employed as probes of evolution; see Riess (2000), Leibundgut (2001), and Perlmutter \& Schmidt (2004) for reviews. The consensus interpretation is that there is no evidence for evolution with limits at or below the statistical constraints on the average high-redshift apparent brightness of SNe Ia. The observed nominal dispersion of high-redshift SNe Ia substantially limits the patchiness of uncorrected extinction, and near-IR observations of a highredshift SN Ia demonstrate that a large opacity from grayish dust is unlikely (Riess et al. 2001). Non-SN constraints on gray dust from QSOs observed in X-rays (Paerels et al. 2002; K. Ninomiya, T. Yaqoob, \& S. Khan 2003, private communication) and a partial or complete resolution of the far-IR background by SCUBA (Chapman et al. 2003) place stringent limits of less than a few percent of dimming at $z=0.5$ from gray dust.

Based on this evidence, we adopt in the following analysis an a priori constraint that the net astrophysical contamination of SN Ia distance measures does not exceed their statistical uncertainty in their mean brightness. Quantitatively, our adopted limit on systematics is defined to be $5 \%$ per $\Delta z$ at $z>0.1$.

First we consider the SN data within an FRW cosmology of unknown curvature and mass density (with a flat prior on all parameters), with the simplest description of a dark energy component (i.e., a cosmological constant) using equation (11). Joint confidence intervals in the $\Omega_{M}-\Omega_{\Lambda}$ plane were derived after numerical integration of the probability density $P\left(H_{0}\right) \propto$ $\exp \left[-\chi^{2}\left(H_{0}\right) / 2\right]$ over all values of $H_{0}$ and are shown in Figure 8. Compared to the same analysis in Riess et al. (1998), the gold sample presented here reduces the area of the $1 \sigma$ contour by a factor of 6 (a factor of 7 including the silver sample). With the current sample, the $4 \sigma$ confidence intervals (i.e., $>99.99 \%$ confidence) are now fully contained within the region where $\Omega_{\Lambda}>0$. The "concordance" model of $\Omega_{M}=$ $0.27, \Omega_{\Lambda}=0.73$ lies within the $1 \sigma$ contour (although just outside of it with the addition of the silver set). For a flatgeometry prior, we measure $\Omega_{M}=0.29 \pm_{0.03}^{0.05}$ (equivalently, $\left.\Omega_{\Lambda}=0.71\right)$. The HST-discovered SNe Ia alone decrease the area of the $1 \sigma$ contour by a factor of 1.5 (in the gold sample) because of their high mean redshift.

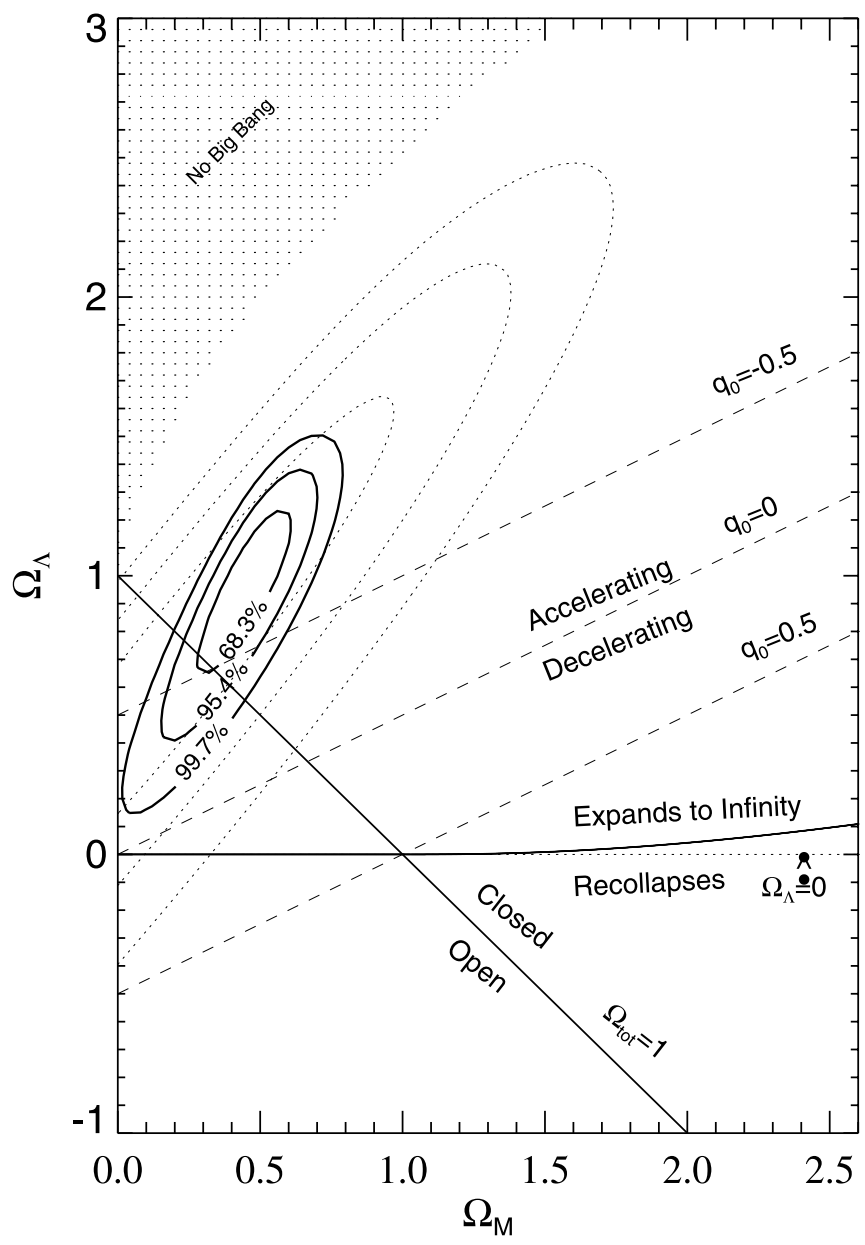

FIG. 8.- Joint confidence intervals for $\left(\Omega_{M}, \Omega_{\Lambda}\right)$ from SNe Ia. The solid contours are results from the gold sample of $157 \mathrm{SNe}$ Ia presented here. The dotted contours are the results from Riess et al. (1998) illustrating the earlier evidence for $\Omega_{\Lambda}>0$. Regions representing specific cosmological scenarios are illustrated. Contours are closed by their intersection with the line $\Omega_{M}=0$.

An alternative approach with good precedent (Garnavich et al. 1998; Perlmutter et al. 1999) is to consider a flat universe and a generalized dark energy component parameterized by its (assumed) constant equation of state $w=P / \rho c^{2}$. Flatness is assumed either on theoretical grounds (i.e., as a consequence of inflation) or on observational grounds from the characteristic angular size scale of the CMB fluctuations (Spergel et al. 2003 and references therein). In this case the luminosity distance is given by

$$
\begin{aligned}
d_{L}= & c H_{0}^{-1}(1+z) \int_{0}^{z} d z\left[(1+z)^{3}\left(\Omega_{M}\right)\right. \\
& \left.+\left(1-\Omega_{M}\right)(1+z)^{3(1+w)}\right]^{-1 / 2} .
\end{aligned}
$$

We determined the probability density in the $\Omega_{M}-w$ plane in the same manner as above, and the results are shown in the left panel of Figure 9. The SN Ia data alone require $w<$ -0.5 for any value of $\Omega_{M}$ at the $95 \%$ confidence level and are consistent with $w=-1$ (i.e., dark energy resembling a cosmological constant) at the $68 \%$ confidence level for $0.20<\Omega_{M}<0.35$. 


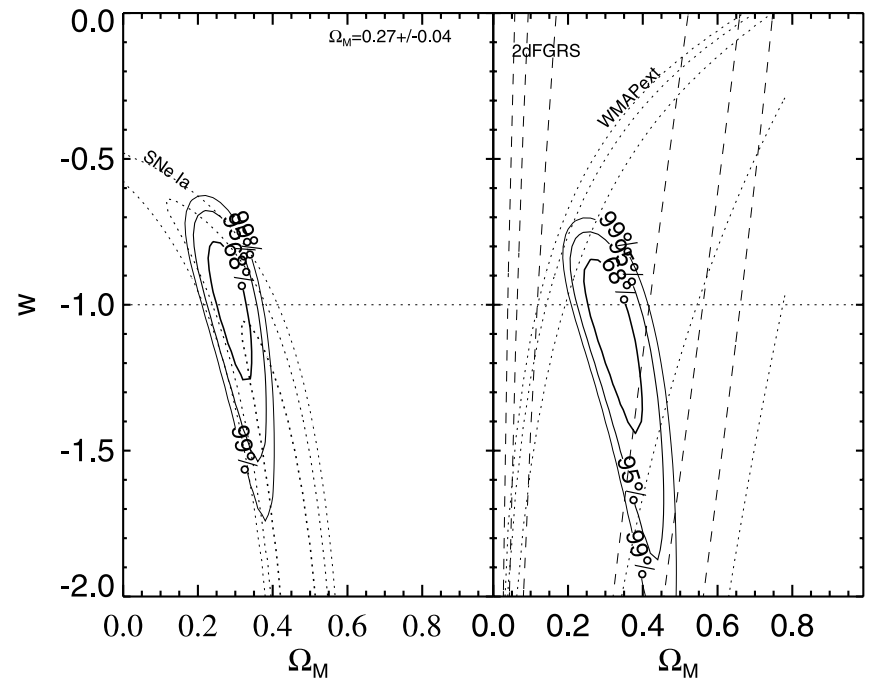

FIG. 9.- Joint confidence intervals for $\Omega_{M}$ and a static equation of state for dark energy, $w$. In the left panel, constraints from the gold SN Ia sample (dotted contours) are combined with a prior of $\Omega_{M}=0.27 \pm 0.04$ to yield the solid contours. In the right panel, the same SN constraints are combined with those from WMAPext and 2dFGRS to yield the solid contours.

Utilizing SN-independent constraints in this plane (primarily to constrain $\Omega_{M}$ ) yields far more precise constraints for $w$ because of the strong degeneracy between $w$ and $\Omega_{M}$ for SNe Ia. In the left panel of Figure 9 we use $\Omega_{M}=0.27 \pm 0.04$ (at $z=0$ ) as a simple approximation to the constraints derived from numerous SN-independent experiments (see Freedman \& Turner 2003 for a review). Alternatively (Fig. 9, right), we used the WMAPext (Bennett et al. 2003; Spergel et al. 2003) measurement of the reduced distance to the surface of last scattering at $z=1089$ and the Two-Degree Field Galaxy Redshift Survey (2dFGRS) measurement of the growth parameter, $f=\left\{\Omega_{M} /\left[\Omega_{M}+\left(1-\Omega_{M}\right)(1+z)^{3 w}\right]\right\}^{0.6}$, to derive independent constraints in the $\Omega_{M}-w$ plane. The results from either approach are similar. We find $w=-1.02 \pm_{0.19}^{0.13}$ and $w=$ $-1.08 \pm_{0.18}^{0.20}$ with the use of an $\Omega_{M}$ prior and from WMAPext + 2 dFGRS, respectively. Using the prior on $\Omega_{M}$, the $95 \%$ confidence interval is $-0.78>w>-1.46$, or $w<-0.76$ if $w \geq$ -1 . The $99 \%$ level constrains $w$ to less than -0.72 . These results are somewhat more constraining than those from Tonry et al. (2003), Barris et al. (2004), and Knop et al. (2003).

The high relative redshifts of the HST-discovered SNe Ia provide little additional power to constrain a $w$ parameter that is fixed a priori to be redshift-independent. The precision of such a constrained study of dark energy is most sensitive to the sheer number of $\mathrm{SNe}$ Ia with a relatively weak dependence on their redshifts (a useful approximation is $\Delta m \approx$ $\left[1.8 z /\left(1+e^{z}\right)\right] \Delta w$ for small $\Delta w, w \approx-1$, and $\left.z<2\right)$ until the systematic error limit is reached.

A more ambitious and potentially more revealing approach to studying dark energy is to allow for both an unconstrained value of the equation of state (at some fiducial redshift, e.g., $z=0$ ) and its time evolution, i.e., $w(z)=w_{0}+w^{\prime} z$, where $\left.w^{\prime} \equiv(d w / d z)\right|_{z=0}$. This parameterization provides the minimum possible resolving power to distinguish a cosmological constant and a rolling scalar field from their time variation (or lack thereof). Indeed, rejection of the hypothesis that $w^{\prime}=0$ would rule out a cosmological constant as the dark energy (as would the determination that $w \neq-1$ ). The measured value of $w^{\prime}$ would provide an estimate of the scale length of a dark energy potential. The only previous estimate of $w^{\prime}$, by Di Pietro \& Claeskens (2003), used the set of SNe Ia from Perlmutter et al. (1999) and the constraints $\Omega_{\text {total }} \equiv 1$ and $\Omega_{M} \equiv 0.3$ and concluded $-12<w^{\prime}<12$ at the $95 \%$ confidence level (best fit: $w_{0}=-1.4, w^{\prime}=2.3$ ).

For $w(z)=w_{0}+w^{\prime} z$, we employ (following Linder 2003)

$$
\begin{aligned}
d_{L}= & c H_{0}^{-1}(1+z) \int_{0}^{z} d z\left[(1+z)^{3}\left(\Omega_{M}\right)\right. \\
& \left.+\left(1-\Omega_{M}\right)(1+z)^{3\left(1+w_{0}-w^{\prime}\right)} e^{3 w^{\prime} z}\right]^{-1 / 2} .
\end{aligned}
$$

A strong degeneracy exists among the three free parameters $w_{0}, w^{\prime}$, and $\Omega_{M}$, requiring the use of independent experimental constraints in this space to make progress. To this end we use the previous prior, $\Omega_{M}=0.27 \pm 0.04$ (at $z=0$ ), which has the advantage of being independent of redshift while providing a good approximation to all non-SN cosmological constraints in this space.

We have avoided using the CMB measurements directly as an additional constraint on the time evolution of the equation of state of dark energy because of difficulties that arise in the analysis of the CMB at $z \gg 1$, where the linear expansion of the epoch-dependent equation of state diverges. Because constraints on $w(z)$ from the CMB are derived from an integration between $z=0$ and $z=1089$, diverging formulations of $w(z)$ are unsuitable. Linder (2003) has proposed a more stable parameterization of $w(z)=w_{0}+w_{a}[z /(1+z)]$; however, for large values of $w_{0}+w_{a}$, which are not rejected by the Di Pietro \& Claeskens (2003) analysis, the CMB integral remains ill-behaved. In addition, the $\mathrm{CMB}$ measurements provide little direct leverage on $w_{a}$ or $w^{\prime}$ compared to SNe Ia at $z \approx 1$ (because $\Omega_{\text {dark energy }} \approx 0$ at $z \gg 1$ ). Therefore, we used the $w^{\prime}$ parameterization, which is well-suited to our SN sample, and a simple prior on $\Omega_{M}$, which avoids problems evaluating functions involving $w(z)$ at $z \gg 1$.

In Figure 10 we show constraints in the $w_{0}-w^{\prime}$ plane (after marginalizing over $\Omega_{M}$ ). Using the gold subset, we find $w_{0}=-1.31 \pm_{0.28}^{0.22}$ and $w^{\prime}=1.48 \pm_{0.90}^{0.81}$, with the uncertainties in both parameters strongly correlated. A cosmological constant (i.e., $w_{0}=-1, w^{\prime}=0$ ) is separated from the best fit along the direction of the major axis of the error ellipse, lying at the boundary of the joint $68 \%$ confidence level. If we constrain the recent behavior of dark energy to be like a cosmological constant (i.e., $w_{0}=-1$ ), we find $w^{\prime}=0.60 \pm$ 0.47 . Models with $w_{0}<-1$ or "phantom energy" (Caldwell et al. 2003) violate the dominant-energy condition $(\rho+p>0)$ and are extremely speculative at this point (and may rip apart the universe in the future), so if we constrain the analysis to $w_{0}>-1$, we find $w^{\prime}=0.6 \pm 0.5$ and $w_{0}<-0.72$ with $95 \%$ confidence. Unfortunately, few theoretical predictions about the size of $w^{\prime}$ exist for dynamic models of dark energy. However, we can reject the possibility that dark energy evolution is currently very rapid (i.e., $\left|w^{\prime}\right|>$ a few). This conclusion alone limits the rate at which simple rolling scalar fields could reach their true minima. The consequences of this statement for predicting the future fate of the universe are discussed in $\S 5$.

Our new constraints in the $w^{\prime}-w$ plane provide a substantial factor of $\sim 8$ improvement over the analysis by Di Pietro \& Claeskens (2003) of the SCP (Perlmutter et al. 1999) data. Still, greater precision for the measurement of $w^{\prime}$ is needed before the proximity (or separation) from $w^{\prime}=0$ could provide a 


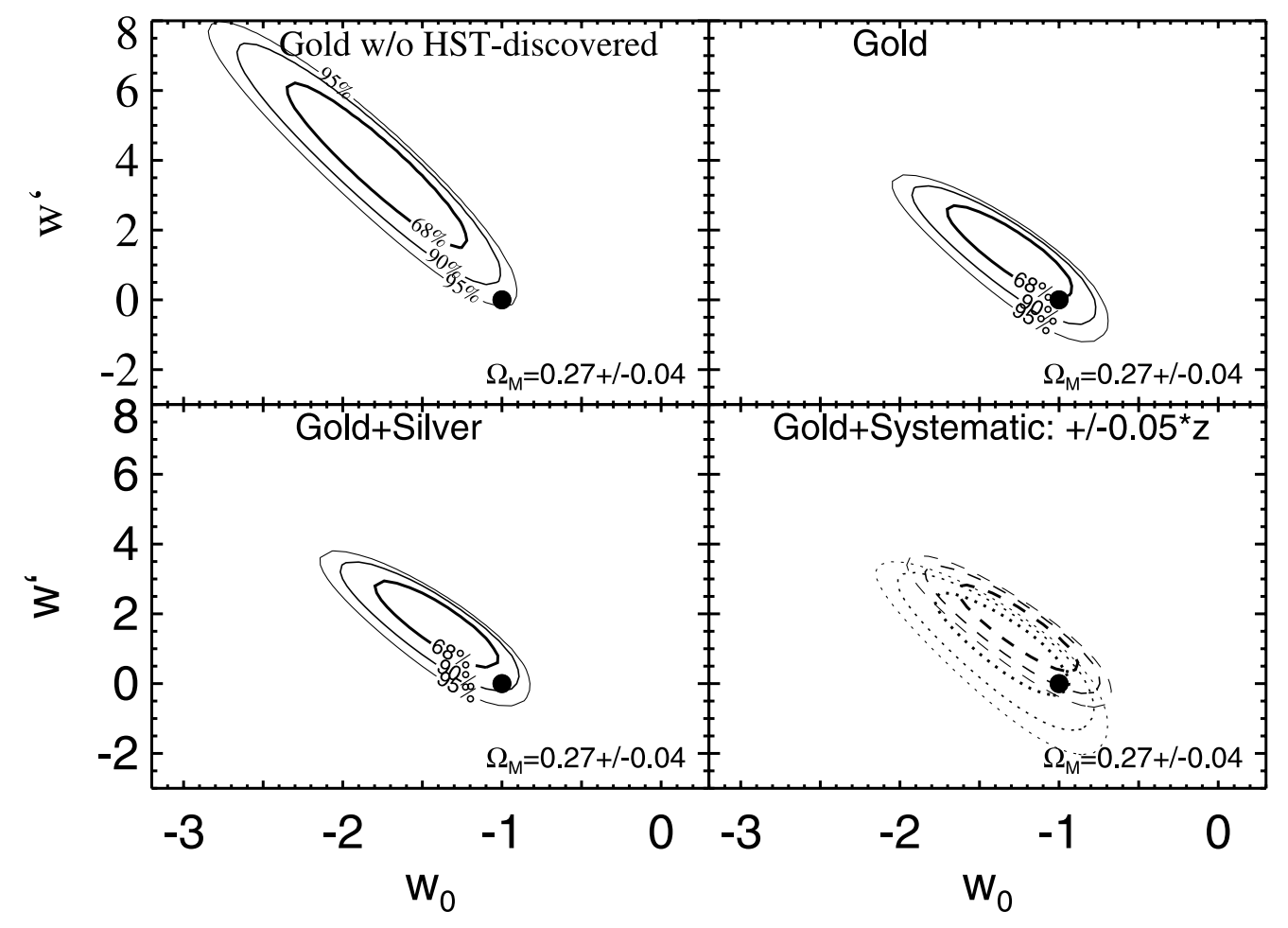

Fig. 10.- Joint confidence intervals derived from SN samples for a two-parameter model of the equation of state of dark energy, $w(z)=w_{0}+w^{\prime} z$. For each panel, constraints from an SN sample are combined with the prior, $\Omega_{M}=0.27 \pm 0.04$, to yield the indicated confidence intervals. The position of a cosmological constant $(-1,0)$ is indicated as a filled symbol. The bottom right panel shows the impact of adding or subtracting a systematic error in distance modulus of $0.05 z$ mag to/from the gold sample.

compelling, empirical case for (or against) a static dark energy (i.e., a cosmological constant). The addition of the silver sample has only a modest impact on this analysis, as seen in Figure 10 (although a cosmological constant crosses to just outside the nominal $68 \%$ confidence interval).

The HST-discovered SNe Ia provide significant leverage in the $w_{0}-w^{\prime}$ plane because of their high mean redshift. Figure 10 (top left) shows the $w^{\prime}-w$ plane without including the HSTdiscovered objects. The HST-discovered SNe Ia alone increase the precision (i.e., reduce the area of the confidence intervals) by an impressive factor of 1.9 , although they account for only $10 \%$ of the sample. Previous studies in support of a dedicated, space-based mission to measure $w_{0}$ and $w^{\prime}$ have concluded that an SN Ia sample must extend to $z>1.5$ to adequately break degeneracies in this parameter space (Linder \& Huterer 2003), a conclusion supported by our analysis. The current relative dearth of SNe Ia at $z>1$ compared to their number at $z<1$ indicates that significant progress can still be made in the constraints on $w^{\prime}$. Proposals for a Supernova/Acceleration Probe (SNAP) or a Joint Dark Energy Mission (JDEM) predict an improved constraint for $w^{\prime}$ over our current analysis by a factor of 3-4, assuming a similar-sized improvement in our knowledge of $\Omega_{M}$ from the Planck satellite and a continued ability to reduce systematic errors (Linder \& Huterer 2003). However, the current sample is rapidly growing in size, and we may expect progress in our constraints on the nature of dark energy in the next few years.

\section{DISCUSSION}

\subsection{Cosmological Constraints}

$\mathrm{SNe}$ Ia at $z>1$ provide valuable and unique contributions to our current understanding of the cosmological model. The current sample of such $\mathrm{SNe}$ Ia, although greatly expanded here, remains small (i.e., <10). Our capacity to constrain simultaneously the full debated range of cosmological and environmental parameters is therefore limited. Consequently, we have chosen to test specific and narrow questions in the context of well-defined assumptions or in conjunction with independent information. It is important to recognize that the conclusions garnered from any analysis cannot furnish a priori information for a subsequent analysis. Readers should carefully consider which priors they are using and where they came from before selecting which analysis presented here provides a relevant incremental gain.

The two most extreme analyses (in the sense of the breadth of their priors) presented here also realize the most significant gains from the addition of our highest redshift SNe Ia discovered with HST. The kinematic (i.e., cause-independent) interpretation of SN Ia distances and redshifts (independent of all other experiments) is most consistent with two distinct epochs of expansion: a recent accelerated expansion and a previous decelerated expansion with a transition between the two at $z \approx 0.5$. This is a generic requirement of a mixed dark matter and dark energy universe, and it may even be a feature of unrelated cosmological paradigms (which are beyond our scope to consider here). The data are not consistent with many astrophysical interpretations posited in lieu of dark energy. Notable examples include the attenuation produced in a universe filled with gray dust at $z>1$, or a luminosity evolution that is a simple, monotonic function of redshift. These interpretations are robust against the exclusion of any individual SN Ia used in the analyses and therefore represent an improvement over the results of Riess et al. (2001).

A vacuum-driven metamorphosis model (VCDM) has been proposed by Parker \& Raval (1999) to explain the cause of 
accelerated expansion. In this model the universe makes a transition to a constant scalar curvature (induced by a quantized noninteracting scalar field of very small mass in its vacuum state) at $z \approx 1$. This model differs from a quintessence model in that the scalar field is free and thus interacts only with the gravitational field. The transition as described by Parker et al. (2003) is far more abrupt than for a cosmological constant and is characterized by $w_{0}=-1.3$ and $w^{\prime}=-0.8$ for values of $\Omega_{M}$ within its likely range. These values lie just outside the $99.5 \%$ confidence level. However, other future variations of the central idea of this model may yield better fits to the data.

The "replenishing dust" model addressed in $\S 4.2$ is one example of a variety of astrophysical dimming models for which most of the apparent dimming occurs by $z \approx 0.5$, with little additional dimming at $z>0.5$. Such models require an additional parameter or a logarithmic parameterization to dampen the dimming and fit the new SNe Ia at higher redshifts. Another model with this behavior would be evolution that is proportional to look-back time (Wright 2001). While possible, such dimming behavior, especially if in the form of luminosity evolution, would seem implausible. We may expect evolution (or dust production) to be coupled to the observed evolution of stellar populations, galaxy morphologies, sizes, large-scale structure, or even chemical enrichment. None of these known varieties of evolution are largely completed by $z=0.5$ starting from their properties at $z=0$; quite the contrary, most of them have hardly begun, looking back to $z=0.5$. A strong empirical argument against recent luminosity evolution is the independence of SN Ia distance measures on gross host morphology (Riess et al. 1998; Sullivan et al. 2003). The range of progenitor formation environments spanned by $\mathrm{SNe}$ Ia in early-type and late-type hosts greatly exceeds any evolution in the mean host properties between $z=0$ and $z=0.5$. In the end, however, the only "proof" against astrophysical contamination of the cosmological signal from $\mathrm{SNe}$ Ia is to test the results against other experiments, independent of SNe Ia.

$\mathrm{SNe}$ Ia are no longer unique in their requirement of a dominant dark-energy component. The bevy of SN-independent cosmological experiments, most notably the CMB, the LSS, and the ISW effect, provide a prior constraint of $\Omega_{\text {total }}=1$ and $\Omega_{M} \approx 0.3$. These priors preserve the limited discriminating power of SN Ia data for resolving the nature of dark energy. What is its equation of state and has it been evolving? The constraints obtained here provide a substantial improvement in our ability to answer the latter (i.e., not rapidly), but the results are far from a compelling, empirical case for a cosmological constant or evolving dark energy.

A parametric reconstruction of $w(z)$ by Alam et al. (2004) of the full set of SN Ia distances from Tonry et al. (2003) and Barris et al. (2004) concluded that dark energy "evolves rapidly" and has "metamorphosized" from $w \sim 0$ at $z \sim 1$ to $w<-1$ at $z \sim 0$. Their analysis differs from ours in a few important ways. Alam et al. (2004) use a significant number of $\mathrm{SNe}$ that would fail our selection criteria of our gold sample, including 26 SNe from the SCP (now excluded or flagged by Knop et al. 2003) and another 13 from Barris et al. (2004) of dubious reliability. Alam et al. (2004) also use a different parameterization for $w(z)$ and slightly different external cosmological constraints. These differences do account for a small part of the difference in our conclusions. However, despite these differences our results are fairly similar to theirs without the inclusion of the new HST-discovered SNe Ia presented here as seen in the top left panel of Figure 10. Without the $H S T$-discovered $\mathrm{SNe}$, the $95 \%$ confidence region resides in the quadrant in which $w_{0}<-1$ and $w(z)$ was less negative in the past. Yet, with the addition of the HST-discovered SNe Ia, the contours shrink in area by a factor of 2 and shift in the direction of a cosmological constant, a position in the $w_{0}-w^{\prime}$ plane that is now at the boundary of the $\sim 1 \sigma$ contour. Such large changes in the size and position of the error contours with the addition of new data indicate the crucial need for even more data before firmer conclusions can be reached.

Knowledge of the equation of state of dark energy and its time evolution has profound implications for determining the fate of the universe. For example, a joint constraint of $w_{0}>-1$ and $w^{\prime}<0$ could provide the signature of a future recollapse (i.e., "big crunch") for a linear potential field that becomes negative in the future (Kallosh \& Linde 2003). Interestingly, the data indicate that this quadrant of the $w_{0}-w^{\prime}$ plane is the least favored. Values (in this quadrant) farthest from the origin $\left(w_{0}=-1\right.$ and $\left.w^{\prime}=0\right)$ would forecast the earliest possible recollapse and are the least favored by our data. Taking the Kallosh \& Linde (2003) toy model at face value, our constraint would rule out such a future recollapse of the universe in less than $\sim 30$ or $15 \mathrm{Gyr}$ at the $95 \%$ or $99 \%$ confidence levels, respectively. This is somewhat more reassuring than the previous minimal remaining time to recollapse of $11 \mathrm{Gyr}$ allowed by the observation that the dark energy density has had time to evolve to $\Omega_{\Lambda} \leq 0.72$ (Kallosh et al. 2003).

A qualitatively different and more speculative fate, a hierarchical "ripping" of progressively smaller bound systems (i.e., "big rip") might occur if $w(z)$ evolves to or remains at values less than -1 (Caldwell et al. 2003). In this case the dark energy density within any bound system would increase without limit as $\rho \propto a^{-3(1+w)}$, overcoming its binding energy. This eventuality may be foretold from joint constraints of $w_{0}<-1$ and $w^{\prime}>0$ and (an assumed) linear extrapolation of the dark energy scalar field. The current data are not inconsistent with this quadrant of the $w_{0}-w^{\prime}$ plane (and will remain consistent if dark energy is a cosmological constant and measurements have finite precision). For an assumed constant equation of state, our $95 \%$ confidence interval would limit a "big rip" to be no sooner than 25 Gyr from now, only slightly more reassuring than the previous estimate of minimal remaining time to the rip of 22 Gyr estimated by Caldwell et al. (2003). However, if $w(z)$ is evolving to progressively more negative values (a result empirically consistent with our data), a rip could occur far sooner. We are limited by our understanding of how the future equation of state of dark energy would track the scale factor, time, or some other global parameter of the universe. Therefore, if dark energy is evolving in this direction, we cannot place any meaningful empirical limit on the minimum time to the rip.

We caution against naively extrapolating our empirical dynamical constraint (fitted over a very small range in redshift) far from the time at which it was derived and is likely to be meaningful. The empirical approach to determining the fate of the universe must rely on large extrapolations until dark energy is better understood.

\subsection{Tests of the Utility of Supernovae}

Many more SNe Ia at $z>1$ may yield a more precise probe of dark energy, provided the statistical power of a larger sample can be realized. It is therefore of interest to use the current set of SNe Ia at $z>1$ to consider whether there are any reasons to believe this goal could not be accomplished. 


\section{SN Ia Composite Light Curves}

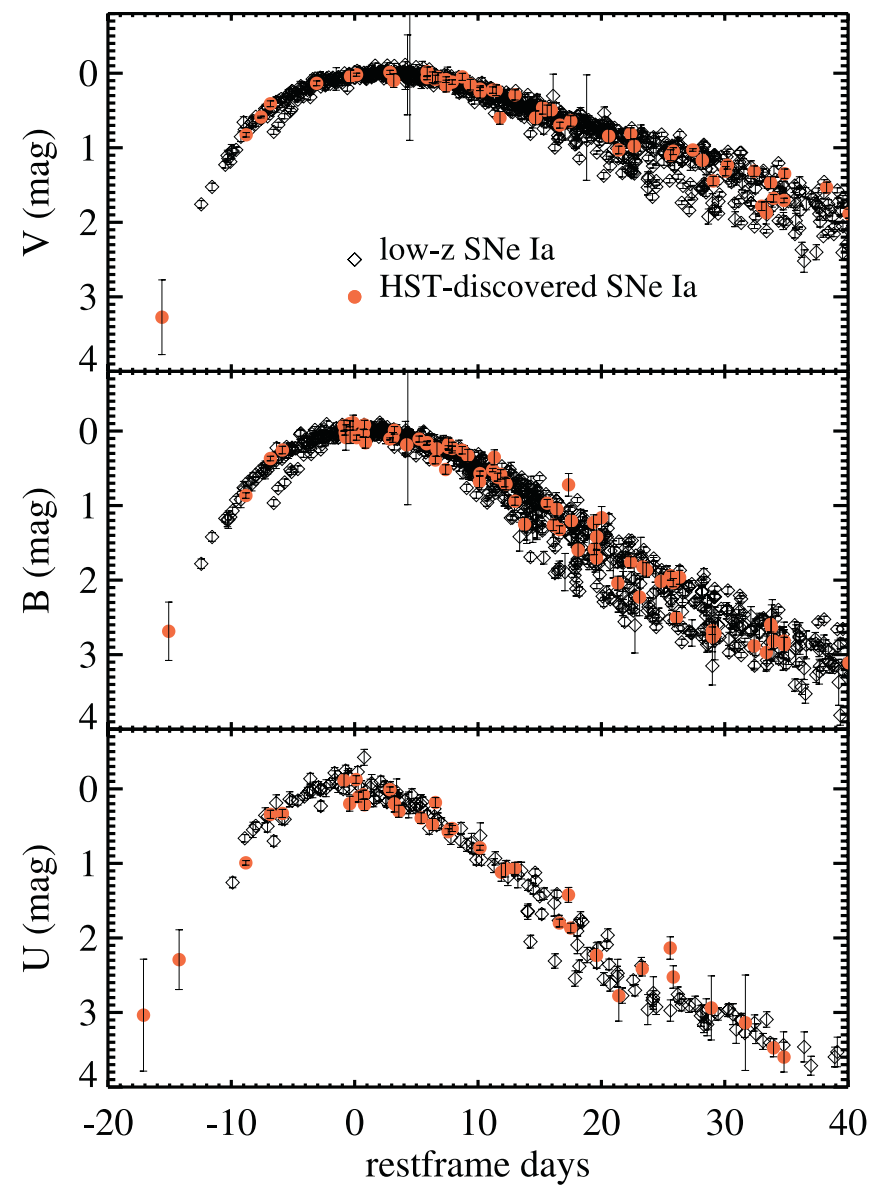

FIG. 11.-Comparison of composite rest-frame light curves of SNe Ia. Each SN Ia is individually transformed to the rest frame ( $K$-corrected; corrected for $1+z$ time dilation). Each is normalized by subtraction of the peak magnitude and its date. Data from SNe Ia with $z<0.1$ are shown as open symbols; data from the HST-discovered SNe Ia are shown as filled symbols.

The dispersion of the SNe Ia at $z>1.0$ around the best-fit model is $0.29 \mathrm{mag}$, similar to the $0.27 \mathrm{mag}$ dispersion of the sample at $0.1<z<1.0$. (We note that these values are higher than the nominal intrinsic dispersion of $\sim 0.15 \mathrm{mag}$ due to the sparse sampling and noisy photometry of SNe Ia observed at high redshifts.) In addition, the average $\chi^{2}$ per $\mathrm{SN}$ at $z>1.0$ ( 9 objects in the gold sample) is 0.95 for the concordance model. We conclude that there is no evidence of excess dispersion for $\mathrm{SNe}$ Ia at $z>1$.

Another test of evolutionary effects is to compare the distribution of light-curve shapes for the HST-discovered SNe Ia with those at low redshift. In Figure 11 we compare the individually normalized (i.e., after subtraction of the individual peak day and apparent magnitude), rest-frame (i.e., after $K$-corrections and correcting for $1+z$ time dilation) light curves of these samples. Statistically, the measured magnitudes of HST-discovered SN light curves (with median redshift 1) are consistent with having been drawn from a parent population at low redshift. The fitted distributions of light-curve shape parameters from the MLCS2k 2 parameterization $\Delta$ (Riess et al. 1996a; S. Jha et al. 2004b, in preparation) are also consistent for the two populations, as shown in Figure 12.

Some evidence for anomalously blue colors of the highredshift SNe Ia in the objects from Riess et al. (1998) was

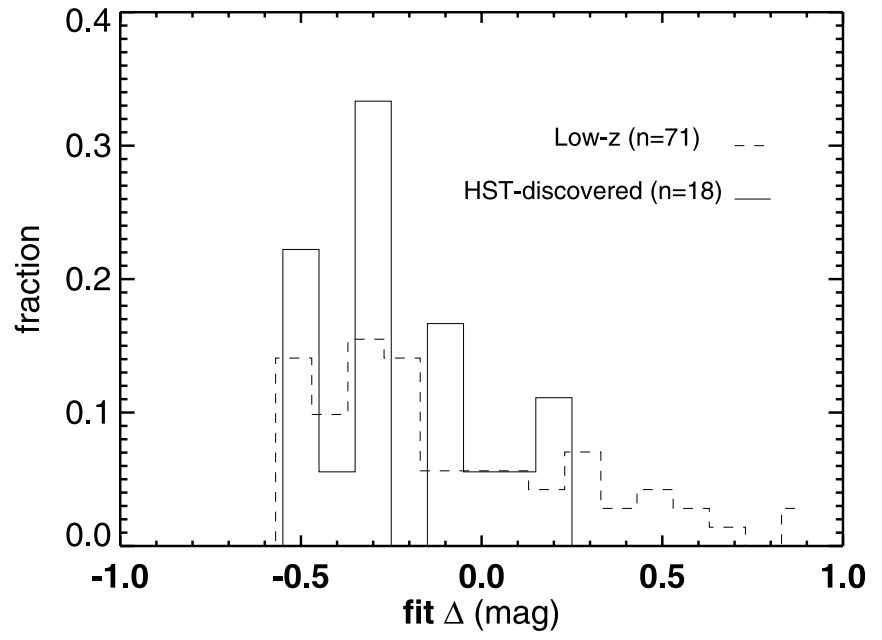

Fig. 12.- - Light-curve shape distributions of low-redshift and high-redshift $\mathrm{SNe}$ Ia. Histograms of the MLCS2k2 parameter $\Delta$ (relative peak visual luminosity) are shown for SNe Ia with $z<0.1$ and the HST-discovered SNe Ia.

noted by Falco et al. (1999) and Leibundgut (2001). The indicated mean difference was a few hundredths of a magnitude in data transformed to rest-frame $B-V$ with a significance approaching $2 \sigma$. No such difference was seen in the data from Perlmutter et al. (1999), although the individual precision of their measured colors may have precluded the detection of such an anomaly. Here we have used the expanded data set and its redshift leverage to look for evidence of color evolution in both rest-frame $B-V$ and $U-B$. The ultraviolet colors of SNe Ia are predicted by theory to be the most sensitive to chemical abundances (Höflich et al. 1998). Figure 13 shows both of these colors as a function of redshift for the sample in a modest range around the unreddened colors of SNe Ia. In Figure 14 we have constructed histograms for the colors in three redshift ranges: $z<0.1,0.1<z<0.6$, and $0.6<z<$ 1.6. For $B-V$, the middle bin is bluer in the mean by $0.02 \mathrm{mag}$ than the lowest bin, and despite the increased sample size the significance of this difference remains $2 \sigma$. This result is not surprising because it makes use of data similar to those of the previous analysis. Interestingly, the size of the mean differences decreases for the highest redshift bin to $0.01 \mathrm{mag}$, with an even lower significance of $1 \sigma$. For the $U-B$ data, the lowest and highest redshift bins are consistent in their means of $-0.45 \pm 0.02$ and $-0.47 \pm 0.02 \mathrm{mag}$, respectively. (Negligible data for $U-B$ in the middle redshift bin is available.) Of the three comparisons presented here, only the previously noted $B-V$ comparison between low and intermediate redshifts is suspect. If the colors of $\mathrm{SNe}$ Ia were evolving, we would expect the comparison at higher redshifts, and additionally with more chemically sensitive colors, to provide amplified evidence for the effect. In addition, the increased sample size in the low- and intermediate-redshift bins has not produced an increase in the significance of the anomaly, as would be expected for a real effect. We therefore conclude there is insufficient evidence for color evolution of $\mathrm{SNe}$ Ia with the current sample, but we encourage future investigators to remain aware of (and test for) this possibility.

Overall, the data presented here suggest that SNe Ia at $z>1$ remain empirically well-behaved and show promise for providing robust cosmological measurements.

To test the sensitivity of our results to the light-curve fitting method used, we compared the MLCS2k2 distances of the 


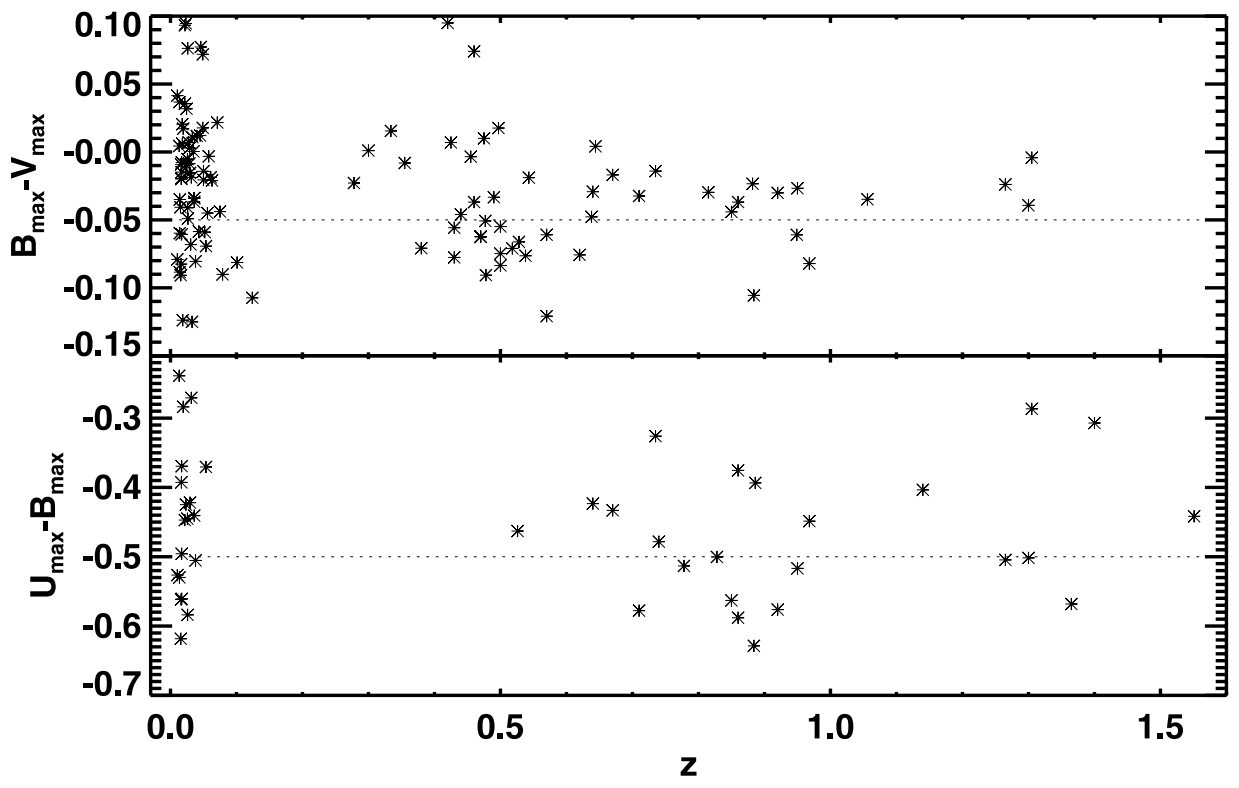

FIG. 13.-Rest-frame $B_{\max }-V_{\max }$ and $U_{\max }-B_{\max }$ colors of SNe Ia vs. redshift. Expected, unreddened colors of SNe Ia are shown as dotted lines.

HST-discovered SNe Ia to those derived from BATM (Tonry et al. 2003). For this comparison, the distance scales of the two methods were normalized by matching the mean distances calculated for the same SNe Ia from Tonry et al. (2003). In Figure 15 we show a comparison for the SNe Ia fitted by both methods over a wide range in redshift. The mean difference (weighted by the quadrature sum of the individual uncertainties) for the HST-discovered SNe Ia is $0.047 \mathrm{mag}$ (in the sense that the BATM distances are larger). This difference is consistent (at the $1 \sigma$ confidence level) with an independent mean uncertainty of $0.18 \mathrm{mag}$ for each method. If we limit the comparison to the nine HST-discovered SNe Ia at $z>0.9$, the mean difference diminishes to less than $0.01 \mathrm{mag}$. Because this higher redshift range provides the leverage utilized for the updated cosmological conclusions presented here, we conclude that our results are insensitive to the light-curve fitting method.

The problem of heterogeneity of SN Ia data may be addressed by future, massive SN surveys such as ESSENCE (Smith et al. 2002; Garnavich et al. 2002) or the CFHT Legacy Survey (Pain et al. 2002), which will obtain large sets of SNe Ia over the full range of explored redshifts (obviating the need for older, heterogeneous data). Until then, we must rely on a careful and judicious compilation of the available data and a clear description of how they were compiled.

Farrah et al. (2004) have used the statistical measurement (most individual hosts were not detected) of submillimeter emission from high-redshift SN Ia hosts to infer the average
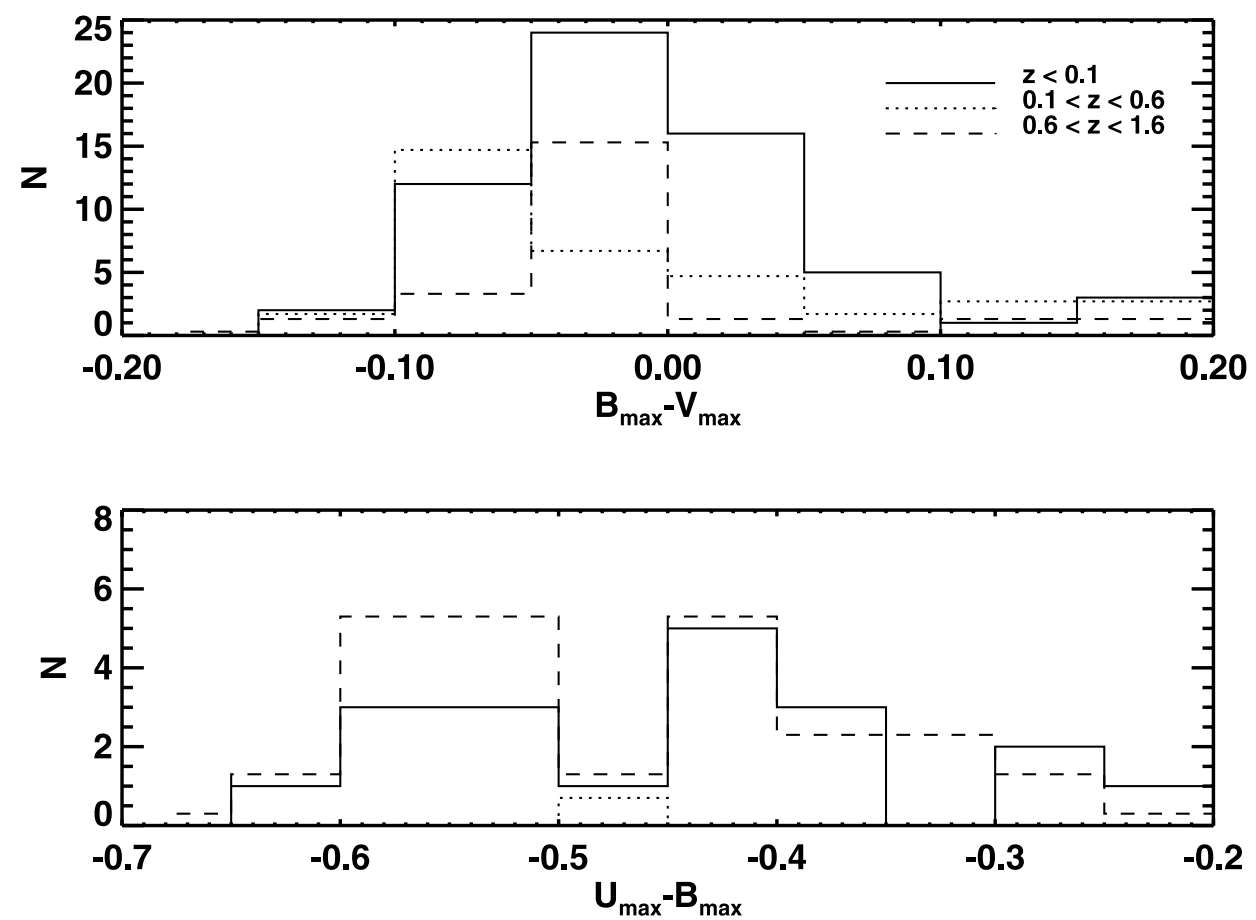

FIG. 14. - Histograms of the color data shown in Fig. 13. The distributions from three different redshift bins are shown as indicated. 


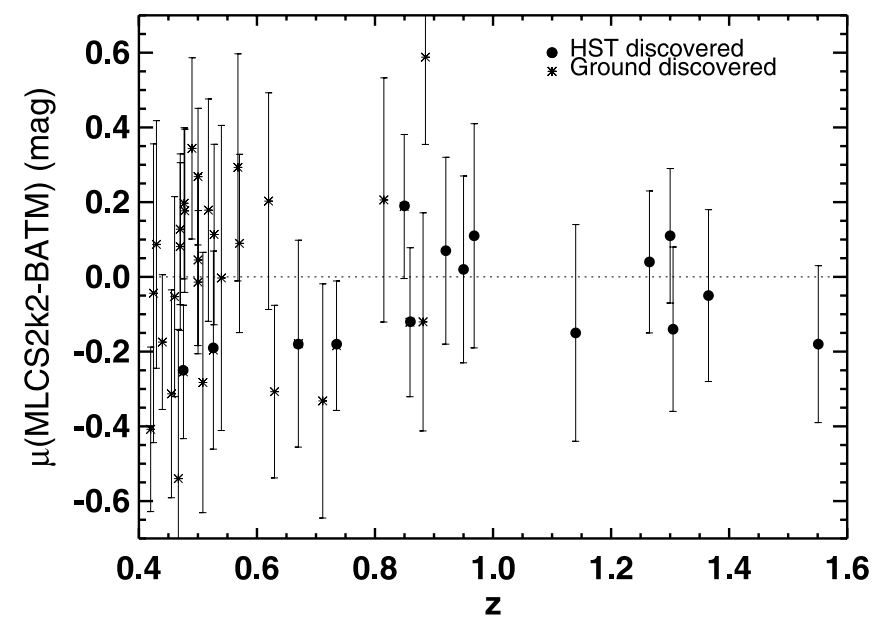

FIG. 15.-Comparison of individual distance differences estimated by the MLCS2k2 and BATM methods for the HST and ground-discovered SNe Ia. The zero points of both methods are normalized by using the same set of SNe Ia.

internal extinction of these galaxies. They then assume that the line-of-sight extinction of supernovae matches the inferred mean extinction of the hosts. They conclude that the SNe Ia from hosts at higher redshifts may suffer more extinction than their lower redshift counterparts and that this effect may bias the inferred cosmology. However, it has not been demonstrated that radio emission correlates well (if at all) with SN extinction. In contrast, apparent reddening has been shown to provide a significant reduction in distance dispersion and should account for any change in the relative proportions of host extinction along the line of sight. More relevant to this work, a trend of increased and uncorrected extinction with redshift does not match the apparent magnitudes of the new $\mathrm{SNe}$ Ia presented here in the highest redshift bin.

\subsection{Lensing}

Until now, we have evaluated the SN data in the context of a homogeneous model for the matter distribution, also known as the "filled beam" model. The inhomogeneity of matter along the SN line of sight distorts spacetime, directing more or less photon-filled lines of sight to our detectors, depending on the matter content along the line of sight. Averaged over enough lines of sight, SN flux is conserved and the inferred cosmology is unaffected. However, further investigation of possible lensing is warranted for small sample sizes and in regard to possible selection biases.

We have estimated the expected lensing along the line of sight of each of the HST-discovered SNe Ia, as well as for 100 randomly selected positions in each of the GOODS fields. The lensing is estimated using the same multiple lens plane methodology employed by Benítez et al. (2002) for SN $1997 \mathrm{ff}$. Photometric and spectroscopic redshifts of all foreground lenses were derived from the GOODS catalogs. Their masses were estimated from the rest-frame $B$-band luminosities and from the Tully-Fisher and Faber-Jackson relations corrected for evolution (Ziegler et al. 2002; Boehm et al. 2004; Kochanek 1996; Treu et al. 2002). Because SN luminosity distances here and elsewhere have been modeled using filledbeam cosmological models, we have calculated the amplifications relative to a filled-beam model and for $\Omega_{M}=0.3$, $\Omega_{\Lambda}=0.7$. The results are shown in Figure 16.

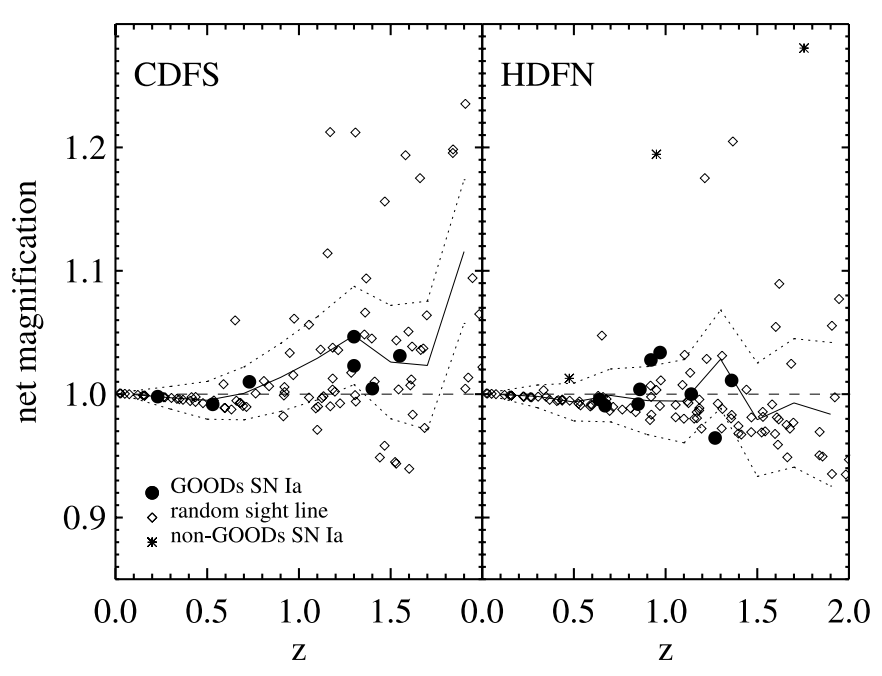

FIG. 16.-Predicted lensing magnifications of $\mathrm{SNe}$ Ia and of random positions in the CDF-S and HDF-N. For the SNe Ia discovered in the GOODS fields, the expected magnification was calculated using a multiple-lens plane formalism, with estimates of foreground lens redshifts and masses derived from the GOODS catalog. Expected magnifications were also calculated for 100 randomly selected positions (redshift and angular position). The solid and dotted lines show redshift bin averages and dispersion, respectively. SNe Ia found in the GOODS survey and in other HST searches are indicated.

As expected, the distribution of net amplifications for random three-dimensional positions in the fields scatters about unity with a width steadily increasing with redshift (simulations predict $\sigma=0.03$ mag per unit redshift as indicated; D. Holz 2003, private communication). Relatively underdense and frequent lines of sight resulting in net deamplification are compensated by an extended tail of strong magnifications, resulting in an increasingly skewed distribution at higher redshifts. These results are in good accord with Monte Carlo simulations of this effect (Holz 1998; Metcalf \& Silk 1999).

We find that the magnification estimates for the SNe Ia discovered in the GOODS survey are consistent with having been drawn from those in the random positions sample. We conclude that there is no evidence of a lensing-induced selection bias of our sample. This is not unexpected since most of our $\mathrm{SNe}$ Ia were discovered at more than 2 mag brighter than the survey limit and lensing would contribute an insignificant $\leq 0.2 \mathrm{mag}$ of amplification for $98 \%$ of the population at $z<1.5$.

Interestingly, there is an indication that the discovery of SNe Ia in the HDF-N found by other surveys could have been favored by amplification. The net amplification of SN $1997 \mathrm{ff}$ of $\sim 0.3$ mag (Benítez et al. 2002) is unusually large, and the SN was within $0.5 \mathrm{mag}$ of its survey limit (Gilliland et al. $1999)$. It is less clear whether the discovery of SN 2002dd $(z=0.95)$ could have been favored by its 0.2 mag amplification because of the serendipity of its discovery (Blakeslee et al. 2003).

In the absence of an apparent selection bias (or bad luck) in our SN survey, it is unnecessary (and even undesirable) to correct individual $\mathrm{SNe}$ for the estimated net amplification, since the sample will approach a mean amplification of unity when considered in a filled-beam model. An important exception would be to correct (or flag) $\mathrm{SNe}$ whose predicted amplification places them on the strong lensing tail, such as SN $1997 \mathrm{ff}$ as done by Benítez et al. (2002), Tonry et al. 


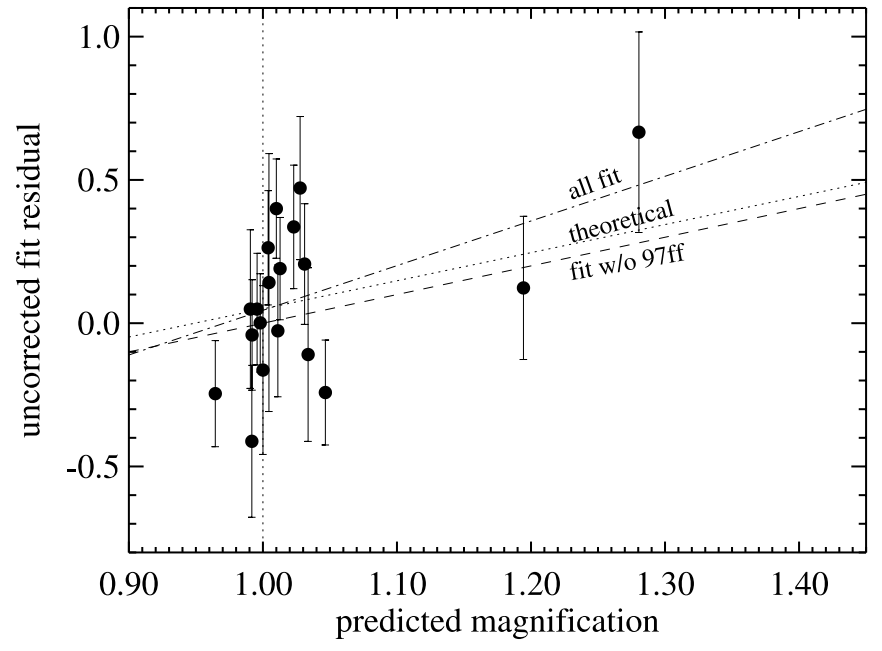

FIG. 17.-Correlation of the predicted magnification and the best-fit cosmological model residual for individual SNe Ia. The predicted magnifications are as described in Fig. 16. The residuals are the difference in distance modulus as predicted from the best-fit model $\left(\Omega_{M}=0.3, \Omega_{\Lambda}=0.7\right)$ and as observed. The empirical correlation is expected to be unity (if the lens light traces their mass) and is shown for the whole sample and without SN 1997ff, the $\mathrm{SN}$ with the largest residual and predicted magnification.

(2003), and here. However, an independent test of this conclusion is available by comparing the predicted amplifications with the observed residuals from a good-fit cosmological model. In Figure 17 we show this comparison and the derived correlation. Empirically we find the residuals to be $1.6 \pm 0.9$ times the predicted magnifications, consistent with the theoretical relation (i.e., unity) and inconsistent with no correlation at the $1.7 \sigma(80 \%)$ confidence level. Much of the leverage comes from SN 1997ff; without it, the correlation is $1.0 \pm 1.2$. As the sample expands, it may soon be possible to provide independent evidence of the correlation of dark matter and light from the high-redshift SN sample.

MGG2001 and more recently Gunnarsson (2004) have made predictions of the expected magnification of two of the $\mathrm{SNe}$ Ia in our sample, both contained in the original HDF-N. For SN 1997ff $(z=1.7)$, these authors concluded that the magnification was uncertain and potentially large (i.e., as high as a factor of a few). In contrast, Benítez et al. (2002) estimated a much more modest magnification of $0.34 \pm 0.12 \mathrm{mag}$, in good agreement with the values estimated by Lewis \& Ibata (2001) and Riess et al. (2001). The source of the potentially higher magnification can be traced to differences in the treatment of the mass scaling of the foreground lenses. MGG2001 treated the lensing galaxies as unevolved with an unknown but possibly high mass scaling (e.g., velocity dispersions of $200-300 \mathrm{~km} \mathrm{~s}^{-1}$ for $M_{*}$ galaxies with $M_{B}=$ $-19.5+5 \log h)$. Benítez et al. (2002) used the $B$-band TullyFisher relation observed by Ziegler et al. (2002) for late-type galaxies at redshifts $0.1-1.0$ to provide a slope and normalization that accounts for evolution (which yields a velocity dispersion $M_{*} \sim 150 \mathrm{~km} \mathrm{~s}^{-1}$ ). The result is a much smaller mass scale than that considered by MGG2001 for the foreground lenses of high-redshift SNe.

For SN 2003es, Gunnarsson (2004) estimated a magnification factor of 1.15 (with a velocity dispersion of $170 \mathrm{~km} \mathrm{~s}^{-1}$ ) for 13 apparent foreground galaxies (at $z<0.968$ ) based primarily on optical photometric redshifts. To span the rest-frame optical breaks in the spectral energy distributions of potential high-redshift foreground galaxies and reliably estimate their redshifts, it is important to use near-IR observations with good precision. We reanalyzed the photometric redshifts of these 13 galaxies using the high-resolution near-IR data from NICMOS (Budavari et al. 2000). In the majority of cases we found that the data from Fernández-Soto et al. (1999) and Gwynn \& Hartwick (1996) contained detections in only three or four bands, whereas the NICMOS data provided a total of five to seven bands and with greater leverage. We found 7 of the 13 galaxies to lie in the background of the SN. Repeating the analysis of Gunnarsson (2004) using their Q-LET algorithm for the seven remaining foreground galaxies reduced the predicted magnification factor to 1.10 . However, calculations from the Q-LET algorithm are made relative to a nonfilled-beam cosmology, and hence all lines of sight are amplified (in the absence of any foreground lenses, the Q-LET amplification would be 1.0). At the redshift of SN 2003es, the average strong-lensing amplification would be $\sim 0.03 \mathrm{mag}$, so the remaining excess would be a factor of 1.07. The remaining difference between this value and our estimate of 1.03 results from the previously discussed difference in the treatment of the size and evolution of the mass scale. Our analysis, comparing the predicted magnification and observed cosmological residuals, is consistent with the mass scaling from Ziegler et al. (2002) as utilized here (and would be inconsistent with a scale approximately twice as large).

It is tempting to consider that we have reached the end of the beginning in the exploration of dark energy. Two reliable and independent routes require it, in addition to a third more tentative investigation via the integrated Sachs-Wolfe effect (Scranton et al. 2003). SNe Ia continue to provide the most direct route to illuminating dark energy because their light can be measured propagating from within its era of dominance. Two clues about dark energy, its equation of state and its recent time evolution, would be invaluable aids to test and provoke theories. We suggest that the most efficient way forward in the near term is by simultaneously mining both ends of the observable redshift range: at $z<1$ generally from the ground and at $z>1$ generally from space. The constraints presented here in the $w_{0}-w^{\prime}$ plane have reduced the allowable range of $w^{\prime}$ from a factor of $\sim 10$ to $\lesssim 1$ while retaining the constraints on $w_{0}$ within $-1.4<w_{0}<-0.7$. With continued determination, an improvement in precision by a factor of a few in this plane is expected.

\section{SUMMARY AND CONCLUSIONS}

We have conducted the first space-based SN search and follow-up campaign using the ACS on board HST. The search parameters and the full list of 42 new SNe are provided elsewhere (L.-G. Strolger et al. 2004, in preparation). We reviewed the sample of SNe Ia harvested from the survey and examined its cosmological significance. The key results can be summarized as follows:

1. We obtained multicolor light curves and spectroscopic redshifts for 16 new SNe Ia that uniformly sample the redshift range $0.2<z<1.6$. Twelve of these are classified by their spectra, two from their red, early-type host galaxies, and two by photometric diagnostics. Three of the SN spectra are at the highest redshifts yet observed for SNe. Six of the SNe Ia are among the seven highest redshift $\mathrm{SNe}$ Ia known; all are at $z>1.25$. These data provide a robust extension of the Hubble diagram to $1<z<1.6$.

2. Utilizing a simple kinematic description of the magnituderedshift data, we find that the $\mathrm{SNe}$ Ia favor recent acceleration 
and past deceleration at the $99.2 \%$ confidence level. An alternate kinematic parameterization requires a positive jerk (third derivative of the scale factor). The best-fit redshift of the transition between these kinematic phases is $z=0.46 \pm 0.13$, although the precise value depends on the kinematic model employed.

3. We have compared the goodness of fit of cosmological models and simple models of astrophysical dimming. The "gold" sample of $157 \mathrm{SNe}$ Ia is consistent with the "cosmic concordance" model $\left(\Omega_{M}=0.3, \Omega_{\Lambda}=0.7\right)$, with $\chi_{\text {dof }}^{2}=1.06$. The data reject at high confidence simple, monotonic models of astrophysical dimming that are tuned to mimic the evidence for acceleration at $z \approx 0.5$. These models include either a universe filled with gray dust at high redshift or luminosity evolution $\propto z$. More complex parameterizations of astrophysical dimming that peak at $z \approx 0.5$ and dissipate at $z>1$ remain consistent with the SN data (but appear unattractive on other grounds).

4. For a flat universe with a cosmological constant, we measure $\Omega_{M}=0.29 \pm_{0.03}^{0.05}$ (equivalently, $\Omega_{\Lambda}=0.71$ ). When combined with external flat-universe constraints, including the CMB and LSS, we find for the dark energy equation of state $w=-1.02 \pm_{0.19}^{0.13}$ (and $w<-0.76$ at the $95 \%$ confidence level) for an assumed static equation of state of dark energy, $P=w \rho c^{2}$.

5. Joint constraints on both the recent equation of state of dark energy and its time evolution are a factor of $\sim 8$ more precise than its first estimate and twice as precise as those derived without the SNe Ia discovered by HST. Both of these dark energy properties are consistent with a cosmological constant (i.e., with $w_{0}=-1.0, w^{\prime}=0$ ) and are inconsistent with very rapid evolution of dark energy (i.e., $\left|w^{\prime}\right|>$ a few). The absence of rapid evolution places constraints on the time in which a simple scalar field could evolve to recollapse the universe. Specifically, the timescale to a potential recollapse is larger than $\sim 30 \mathrm{Gyr}$. If dark energy is evolving toward more negative $w$, we cannot place any meaningful limit on the minimum time to a (speculative) big rip.

We wish to thank Richard Hook, Swara Ravindranath, Tomas Dahlen, Peter Garnavich, Duilia de Mello, Ed Taylor, Soo Kim, Rafal Idzi, Carl Biagetti, Lexi Moustakas, Marin Richardson, Vicki Laidler, Ann Hornschmeier, Ray Lucas, Norman Grogin, Claudia Kretchmer, Brian Schmidt, Stephane Blondin, and Anton Koekemoer for their help in the supernova search. We are grateful to Dorothy Fraquelli, Sid Parsons, Al Holm, Tracy Ellis, Richard Arquilla, and Mark Kochte for their help in assuring rapid delivery of the data. We thank Tom Matheson, Dan Stern, Hy Spinrad, Piero Rosati, Mario Nonino, Alice Shapley, Max Pettini, and Dawn Erb for their efforts to obtain redshifts of some SN host galaxies. We appreciate the guidance of Anton Koekemoer and Eddie Bergeron. This work is partly based on observations collected at the European Southern Observatory, Chile (program 70.A-0497). Financial support for this work was provided by NASA through programs GO-9352 and GO-9583 from the Space Telescope Science Institute, which is operated by AURA, Inc., under NASA contract NAS 5-26555. Some of the data presented herein were obtained at the W. M. Keck Observatory, which is operated as a scientific partnership among the California Institute of Technology, the University of California, and NASA; the Observatory was made possible by the generous financial support of the W. M. Keck Foundation.

\section{APPENDIX}

\section{THE FULL SAMPLE}

Distance measurements to individual SNe depend on the algorithms used to estimate their $K$-corrections, fit their light curves, and infer their extinction. There is currently no single set of algorithms that are considered by consensus to be the optimal ones. Rather, different methods may have advantages depending on the breadth and quality of the observational record of any individual SN Ia. In addition, algorithms improve as their training samples grow. Here we present the full cosmological sample of SNe Ia used in this work in Table 5. Their virtue is that all distance estimates were derived from a single set of algorithms, MLCS2k2 (S. Jha et al. 2004a, in preparation), with the broadest set of training data available at this time, including $U$-band data. Additional advantages

TABLE 5

MLCS2k2 Full SAMPLE

\begin{tabular}{|c|c|c|c|c|c|}
\hline $\mathrm{SN}$ & $z$ & $\mu_{0}{ }^{\mathrm{a}}$ & $\sigma^{\mathrm{b}}$ & Host $A_{V}$ & Sample \\
\hline SN 1990T........................... & 0.0400 & 36.38 & 0.19 & 0.37 & Gold \\
\hline SN 1990af.......................... & 0.050 & 36.84 & 0.21 & -0.04 & Gold \\
\hline SN 19900 & 0.0307 & 35.90 & 0.20 & 0.11 & Gold \\
\hline SN 1991S.......................... & 0.0560 & 37.31 & 0.18 & 0.20 & Gold \\
\hline SN 1991U ....................... & 0.0331 & 35.54 & 0.20 & 0.37 & Gold \\
\hline 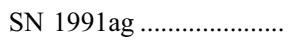 & 0.0141 & 34.13 & 0.25 & 0.12 & Gold \\
\hline SN 1992J.. & 0.0460 & 36.35 & 0.21 & 0.25 & Gold \\
\hline SN 1992P.. & 0.0265 & 35.64 & 0.20 & 0.17 & Gold \\
\hline 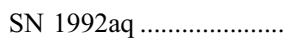 & 0.101 & 38.73 & 0.20 & -0.03 & Gold \\
\hline 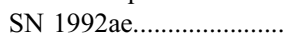 & 0.075 & 37.77 & 0.19 & 0.16 & Gold \\
\hline 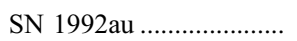 & 0.061 & 37.30 & 0.22 & 0.09 & Gold \\
\hline
\end{tabular}

Note-Table 5 is published in its entirety in the electronic edition of the Astrophysical Journal. A portion is shown here for guidance regarding its form and content.

a Distance normalization is arbitrary; see Appendix.

b Peculiar velocity of $400 \mathrm{~km} \mathrm{~s}^{-1}$ included for all SNe and $2500 \mathrm{~km} \mathrm{~s}^{-1}$ if $z$ derived from SN. 
include a consistent and thorough reanalysis of quality criteria for all currently published $\mathrm{SNe}$ Ia, an exercise resulting in the rejection of many SNe from our "gold" sample whose observational records have one or more shortcomings (see $\S 3.1$ for discussion).

The zero point, distance scale, absolute magnitude of the fiducial SN Ia, and Hubble constant derived from Table 5 are all closely related (or even equivalent) quantities that were arbitrarily set for the sample presented here. Their correct value is not relevant for the analyses presented, which only make use of differences between SN Ia magnitudes. Thus the analyses are independent of the aforementioned normalization parameters.

Aguirre, A. N. 1999a, ApJ, 512, L19 1999b, ApJ, 525, 583

Alam, U., Sahni, V., \& Starobinsky, A. A. 2004, preprint (astro-ph/0403687)

Alard, C., \& Lupton, R. H. 1998, ApJ, 503, 325

Barris, B., et al. 2004, ApJ, 602, 571

Benítez,N., Riess, A., Nugent,P., Dickinson, M., Chornock, R., \& Filippenko, A.V. 2002, ApJ, 577, L1

Bennett, C., et al. 2003, ApJS, 148, 1

Blakeslee, J. P., et al. 2003, ApJ, 589, 693

Boehm, A., et al. 2004, A\&A, in press

Budavári, T., Szalay, A. S., Connolly, A. J., Csabai, I., \& Dickinson, M. 2000, AJ, 120, 1588

Caldwell, R. R., Davé, R., \& Steinhardt, P. J. 1998, Ap\&SS, 261, 303

Caldwell, R. R., Kamionkowski, M., \& Weinberg, N. N. 2003, Phys. Rev. Lett., 91, 71301

Carroll, S. M., Press, W. H., \& Turner, E. L. 1992, ARA\&A, 30, 499

Chapman, S. C., Blain, A. W., Ivison, R. J., \& Smail, I. 2003, Nature, 422, 695

Coil, A. L., et al. 2000, ApJ, 544, L111

Colgate, S. 1979, ApJ, 232, 404

Cowie, L. L., Barger, A. J., Hu, E. M., Capak, P., \& Songaila, A. 2004, AJ, submitted (astro-ph/0401354)

Deffayet, C., Dvali, G., \& Gabadadze, G. 2002, Phys. Rev. D, 65, 044023

Di Pietro, E., \& Claeskens, J. 2003, MNRAS, 341, 1299

Drell, P. S., Loredo, T. J., \& Wasserman, I. 2000, ApJ, 530, 593

Falco, E., et al. 1999, ApJ, 523, 617

Farrah, D., Fox, M., Rowan-Robinson, M., Clements, D., \& Afonso, J. 2004, ApJ, 603, 489

Fernández-Soto, A., Lanzetta, K. M., \& Yahil, A. 1999, ApJ, 513, 34

Filippenko, A. V. 1997, ARA\&A, 35, 309 2001, PASP, 113, 1441

2002, in From Twilight to Highlight: The Physics of Supernovae, ed. W. Hillebrandt \& B. Leibundgut (Berlin: Springer), 171

. 2004, in Measuring and Modeling the Universe, ed. W. L. Freedman (Cambridge: Cambridge Univ. Press), in press

Filippenko, A. V., Li, W., Treffers, R. R., \& Modjaz, M. 2001, in IAU Colloq. 183, Small-Telescope Astronomy on Global Scales, ed. W. P. Chen, C. Lemme, \& B. Paczyński (ASP Conf. Ser. 246; San Francisco: ASP), 121

Filippenko, A. V., \& Riess, A. G. 2001, in AIP Conf. Proc. 540, Particle Physics and Cosmology: Second Tropical Workshop, ed. J. F. Nieves (New York: AIP), 227

Freedman, W., \& Turner, M. 2003, Rev. Mod. Phys., 75, 1433

Fruchter, A., \& Hook, R. 1997, Proc. SPIE, 3164, 120

Garnavich, P. M., et al. 1998, ApJ, 509, 74

. 2002, BAAS, 34, 1233

Giavalisco, M., et al. 2004, ApJ, 600, L93

Gilliland, R. L., Nugent, P. E., \& Phillips, M. M. 1999, ApJ, 521, 30

Gilliland, R. L., \& Riess, A. G. 2002, in HST Calibration Workshop, ed. S. Arribas, A. Koekemoer, \& B. Whitmore (Baltimore: STScI), 61

Goobar, A., Bergstrom, L., \& Mortsell, E. 2002, A\&A, 384, 1

Gunnarsson, C. 2004, J. Cosmology Astropart. Phys., 3, 2

Gwynn, S. D. J., \& Hartwick, F. D. A. 1996, ApJ, 468, L77

Hamuy, M., Phillips, M. M., Maza, J., Suntzeff, N. B., Schommer, R. A., \& Aviles, R. 1996a, AJ, 112, 2391

. 1996b, AJ, 112, 2398

Hamuy, M., Trager, S. C., Pinto, P. A., Phillips, M. M., Schommer, R. A., Ivanov, V., \& Suntzeff, N. B. 2000, AJ, 120, 1479
REFERENCES

Hatano, K., Branch, D., \& Deaton, J. 1998, ApJ, 502, 177

Höflich, P., Wheeler, J. C., \& Thielemann, F. K. 1998, ApJ, 495, 617

Holz, D. E. 1998, ApJ, 506, L1

Jha, S. 2002, Ph.D. thesis, Harvard Univ.

Kallosh, R., Kratochvil, J., Linde, A., Linder, E. V., \& Shmakova, M. 2003,

J. Cosmology Astropart. Phys., 10, 15

Kallosh, R., \& Linde, A. 2003, J. Cosmology Astropart. Phys., 2, 2

Knop, R., et al. 2003, ApJ, 598, 102

Kochanek, C. S. 1996, ApJ, 473, 595

Leibundgut, B. 2001, ARA\&A, 39, 67

Lewis, G. F., \& Ibata, R. A. 2001, MNRAS, 324, L25

Linder, E. V. 2003, Phys. Rev. Lett., 90, 91301

Linder, E. V., \& Huterer, D. 2003, Phys. Rev. D, 67, 81303

McLean, I. S., et al. 1998, Proc. SPIE, 3354, 566

Metcalf, R. B., \& Silk, J. 1999, ApJ, 519, L1

Mortsell, E., Gunnarsson, C., \& Goobar, A. 2001, ApJ, 561, 106 (MGG2001)

Nugent, P., Kim, A., \& Perlmutter, S. 2002, PASP, 114, 803

Oke, J. B., \& Sandage, A. 1968, ApJ, 154, 21

Oke, J. B., et al. 1995, PASP, 107, 375

Paerels, F., Petric, A., Telis, G., \& Helfand, D. J. 2002, BAAS, 34, 1264

Pain, R., et al. 2002, BAAS, 34, 1169

Parker, L., Komp, W., \& Vanzella, D. 2003, ApJ, 588, 663

Parker, L., \& Raval, A. 1999, Phys. Rev. D, 60, 123502

Peebles, P. J., \& Ratra, B. 2003, Rev. Mod. Phys., 75, 559

Perlmutter, S., \& Schmidt, B. P. 2004, in Supernovae and Gamma-Ray Bursts, ed. K. Weiler (New York: Springer), in press

Perlmutter, S., et al. 1997, ApJ, 483, 565

. 1999, ApJ, 517, 565

Rana, N. C. 1979, Ap\&SS, 66, 173 1980, Ap\&SS, 71, 123

Riess, A. G. 2000, PASP, 112, 1284

Riess, A. G., Press, W. H., \& Kirshner, R. P. 1995, ApJ, 438, L17 1996a, ApJ, 473, 88 1996b, ApJ, 473, 588

Riess, A. G., et al. 1998, AJ, 116, 1009

1999a, AJ, 117, 707

1999b, AJ, 118, 2675

2001, ApJ, 560, 49

2004, ApJ, 600, L163

Schlegel, D. J., Finkbeiner, D. P., \& Davis, M. 1998, ApJ, 500, 525

Schmidt, B. P., et al. 1998, ApJ, 507, 46

Scranton, R., et al. 2003, Phys. Rev. Lett., submitted (astro-ph/0307335)

Smith, R. C., et al. 2002, BAAS, 34, 1232

Spergel, D. N., et al. 2003, ApJS, 148, 175

Sullivan, M., et al. 2003, MNRAS, 340, 1057

Tammann, G. A. 1979, in ESA/ESO Workshop on Astronomical Uses of the Space Telescope, ed. F. Macchetto, F. Pacini, \& M. Tarenghi (Geneva: ESO), 329

Tonry, J. T., et al. 2003, ApJ, 594, 1

Treu, T., Stiavelli, M., Casertano, S., Møller, P., \& Bertin, G. 2002, ApJ, 564, L13

Turner, M., \& Riess, A. G. 2002, ApJ, 569, 18

Visser, M. 2003, preprint (gr-qc/0309109)

Wirth, G. D., et al. 2004, AJ, submitted (astro-ph/0401353)

Wright, E. L. 2001, BAAS, 34, 574

Ziegler, B. L., et al. 2002, ApJ, 564, L69 